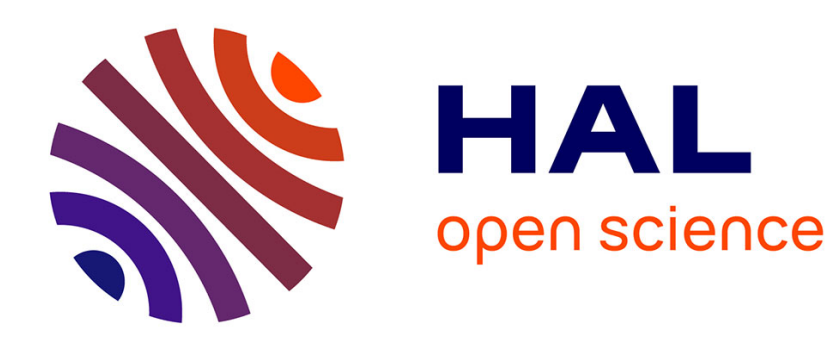

\title{
VARIATIONS ON GROMOV'S OPEN-DENSE ORBIT THEOREM
}

\author{
Charles Frances
}

\section{To cite this version:}

Charles Frances. VARIATIONS ON GROMOV'S OPEN-DENSE ORBIT THEOREM. Bulletin de la société mathématique de France, 2018, 146 (4), pp.713-744. 10.24033/bsmf.2773 hal-02135237

\section{HAL Id: hal-02135237 \\ https://hal.science/hal-02135237}

Submitted on 21 May 2019

HAL is a multi-disciplinary open access archive for the deposit and dissemination of scientific research documents, whether they are published or not. The documents may come from teaching and research institutions in France or abroad, or from public or private research centers.
L'archive ouverte pluridisciplinaire HAL, est destinée au dépôt et à la diffusion de documents scientifiques de niveau recherche, publiés ou non, émanant des établissements d'enseignement et de recherche français ou étrangers, des laboratoires publics ou privés. 


\title{
VARIATIONS ON GROMOV'S OPEN-DENSE ORBIT THEOREM
}

\author{
By Charles Frances
}

\begin{abstract}
We investigate under which conditions a geometric structure which is locally homogeneous on a dense open set is locally homogeneous everywhere. In the case of 3-dimensional Lorentz metric, this allows us to sharpen the conclusions in Gromov's open-dense orbit theorem.

RÉsumÉ. - Nous étudions sous quelles conditions une structure géométrique qui est localement homogène sur un ouvert dense est localement homogène partout. Dans la cadre des variétés lorentziennes de dimension 3, cela conduit à un renforcement des conclusions dans le théorème de l'orbite dense-ouverte de Gromov.
\end{abstract}

\section{Introduction}

The main motivation of this article comes from the following result of $\mathrm{M}$. Gromov, often quoted in the litterature as the open-dense orbit theorem.

Theorem 1.1 ([Gr], Th. 3.3.A). — Let $M$ be a smooth manifold, and $\mathcal{S}$ a smooth rigid geometric structure of algebraic type on $M$. If the automorphism group of $(M, \mathcal{S})$ has a dense orbit, then the structure $\mathcal{S}$ is locally homogeneous on a dense open subset of $M$.

Recall that a structure is locally homogeneous if given any pair of points $(x, y)$ of $M$, there exists a local isometry $f$ (namely a local diffeomorphism preserving $\mathcal{S}$ ), defined from a neighborhood of $x$ to a neighborhood of $y$, and satisfying $f(x)=y$.

Charles Frances, IRMA, 7 rue René Descartes, 67000 Strasbourg.

E-mail : cfrances@math.unistra.fr

2000 Mathematics Subject Classification. - 53C10, 53C23, 53C50. 
The notion of rigid geometric structure of algebraic type was introduced in $[\mathrm{Gr}]$. It covers a wide range of structures, important examples of which are pseudo-Riemannian metrics on manifolds, or affine connections.

Theorem 1.1 actually holds under the weaker assumption that the pseudogroup of local isometries has a dense orbit. It comes as a corollary of a more general result, also proved in [Gr], stating that for a rigid geometric structure of algebraic type, there exists a dense open subset where the orbits of the pseudogroup of local isometries are closed submanifolds. It is thus clear that whenever one of these orbits is dense, it must be open. The reader wanting to learn more about Gromov's theory of rigid transformation groups will find details in [Gr], [DaG], [B], [Z1].

A beautiful application of Theorem 1.1 can be found in [BFL], where the authors use Gromov's result to get a full classification of contact Anosov flows on compact manifolds, admitting smooth stable and unstable distributions. Their strategy is to show that such contact flows preserve a smooth pseudoRiemannian metric, which turns out to be locally homogeneous because of Gromov's theorem, and the Anosov dynamics. The end (actually the main part) of the proof consists in classifying the possible algebraic local models. More generally, Theorem 1.1 seems to be a key ingredient in classifiying rigid geometric structures of a certain type, with a topologically transtive group of isometries. There is however a restriction : the local homogeneity ensured by the theorem is only available on a dense open subset of the manifold, while we would like such a result to hold on the whole manifold. This raises the following natural question :

Question 1.2. - Can the maximal open set of local homogeneity given by Theorem 1.1 be a strict open subset of $M$ ?

While it is expected that the answer to the previous question should be negative, there are very few instances where one can prove it (see [BFL] and [DG] for nontrivial examples where the authors show local homogeneity everywhere).

\subsection{Open-dense orbit theorem and 3-dimensional Lorentz metrics.}

- If we restrict our attention to pseudo-Riemannian structures, the situation seems to be the following. The answer to Question 1.2 is negative for Riemannian manifolds, and pseudo-Riemannian surfaces. In the first case, it is almost obvious, and in the second one, we see that whenever the isometry group has a dense orbit, the sectional curvature must be constant, which implies local homogeneity.

One aim of this paper is to study the first nontrivial case beside the two preceding ones, namely that of 3-dimensional Lorentz manifolds. Our main result is : 
TheOREM A. - Let $\left(M^{3}, g\right)$ be a smooth closed 3-dimensional Lorentz manifold. If the isometry group $\operatorname{Iso}(M, g)$ has a dense orbit, then $\left(M^{3}, g\right)$ is locally homogeneous.

Observe that we don't make any a priori assumption on the group $\operatorname{Iso}(M, g)$. In particular, it might be a discrete group.

Under stronger assumptions, Theorem A can be deduced from previous works. For instance, if we assume that the metric $g$ is real analytic and the manifold $M$ is compact, then S. Dumitrescu showed in [D2] that the existence of a nonempty open orbit for the pseudo-group of local isometries led to local homogeneity.

In the smooth category, under the stronger assumption that there exists a 1-parameter flow of isometries with a dense orbit, Theorem A can be derived from [Z2], where A. Zeghib classifies completely all Lorentzian flows on compact 3-manifolds which are not equicontinuous.

Let us finally mention that obtaining a generalization of Theorem A to Lorentz manifolds of arbitrary dimension, or to general pseudo-Riemannian structures seems to be rather challenging. Good examples of topologically transitive pseudo-Riemannian flows, illustrating the conclusions of Theorem 1.1 , can be built as follows. Let $G$ be a noncompact simple (or semi-simple) Lie group, and let $\Gamma$ be a uniform lattice. Let $\left\{g^{t}\right\}$ be a 1-parameter subgroup of $G$, whith noncompact closure in $G$. It follows from Moore's theorem that $\left\{g^{t}\right\}$ acts ergodically on $G / \Gamma$. Let $\kappa_{0}$ be the Killing form on $\mathfrak{g}$. This is a pseudo-Riemannian scalar product, which is $\operatorname{Ad}\left(g^{t}\right)$-invariant. Pushing this scalar product by right translations, one gets a bi-invariant pseudoRiemannian metric on $G$, which in turns induces a $g^{t}$-invariant metric $h_{0}$ on $G / \Gamma$. This Killing metric $h_{0}$ is actually $G$-invariant, hence homogeneous. The point is that for many 1-parameter groups $\left\{g^{t}\right\}$ (for instance when $\left\{g^{t}\right\}$ is in a Cartan subgroup $A$, or when $\left\{g^{t}\right\}$ is unipotent), there are a lot of $\operatorname{Ad}\left(g^{t}\right)$ invariant pseudo-Riemannian scalar products $\kappa$ on $\mathfrak{g}$ beside the Killing form. Actually, one can choose some $\kappa$ 's which are $\operatorname{Ad}\left(g^{t}\right)$-invariant, without being $\operatorname{Ad}(H)$-invariant for $\left\{g^{t}\right\} \subsetneq H$. By the same construction as above, $\kappa$ yields a pseudo-Riemannian metric $h$ on $G / \Gamma$ for which the isometry group reduces to $\left\{g^{t}\right\}$ (maybe up to finite index). Such a metric is of course no longer homogeneous, but is still locally homogeneous. Now even for those concrete examples, and besides particular choices of groups $\left\{g^{t}\right\}$, it does not seem obvious to show that all pseudo-Riemannian metrics on $G / \Gamma$ which are $\left\{g^{t}\right\}$-invariant are locally homogeneous (a generalization of the results of [Io] might be usefull to this regard).

1.2. Quasihomogeneity. - Let us now discuss purely local problems related to Question 1.2. 
We recall that a local Killing field for a geometric structure $\mathcal{S}$ on a manifold $M$ is a vector field $X$ defined on some open set $U \subset M$, and such that the local flow $\varphi_{X}^{t}$ preserves $\mathcal{S}$. The set of Killing fields defined on $U$ is a Lie subalgebra of the vector fields on $U$, that we denote $\operatorname{kill}(U)$. When the structure $\mathcal{S}$ is rigid, then $\mathfrak{k i l l}(U)$ is always finite dimensional. It then follows that if $\left(U_{i}\right)_{i \in \mathbb{N}}$ is a nested family of open sets containing a point $x$, and satisfying $\bigcap_{i \in \mathbb{N}} U_{i}=\{x\}$, then the dimension of $\mathfrak{k i l l}\left(U_{i}\right)$ stabilizes for $i$ large enough. The resulting Lie algebra will be denoted by $\mathfrak{k i l r}(x)$.

Starting from a point $x \in M$, we can consider the set of all points $y \in M$ that can be reached from $x$ by flowing along successive local Killing fields. This set is called the Kill ${ }^{\text {loc }}$-orbit of $x$, and denoted $\mathcal{O}_{x}^{\text {loc }}$. Let us recall that for "generic" rigid structures, there are no local Killing fields at all, and the Kill ${ }^{\text {loc }}$-orbits are reduced to points. The opposite situation is that of connected locally homogeneous structures, for which $\mathcal{O}_{x}^{\text {loc }}=M$. An interesting weaker notion is that of quasihomogeneous structure.

Definition 1.3. - A geometric structure is called quasihomogeneous when the union of open Killoc ${ }^{\mathrm{oc}}$ orbits is dense.

Gromov's theorem 1.1 says that a rigid geometric structure of algebraic type, with a topologically transitive automophism group, is quasihomogeneous. It is thus a question of general interest to understand when a quasihomogeneous structure is actually locally homogeneous.

It seems that there is no universal answer to this problem. For instance, A. Guillot and S. Dumitrescu exhibited in [DG] quasihomogeneous affine connections on surfaces which are not homogeneous, even in the real analytic category.

On the contrary, S. Dumitrescu and K. Melnick recently showed in [DM] that any real analytic Lorentz metric on a 3-manifold which is quasihomogeneous must be locally homogeneous. The analyticity assumption is crucial in their proof, and it is unknown if the results of [DM] still hold in the smooth category.

Actually, Theorem A will follow from a partial generalization of $[\mathrm{DM}]$ to smooth manifolds. We will indeed show the following local result :

Theorem B. - Let $\left(M^{3}, g\right)$ be a smooth 3-dimensional Lorentz manifold (not necessarily closed). Assume that on a dense open subset, the Lie algebra of local Killing fields is at least 4-dimensional. Then $\left(M^{3}, g\right)$ is locally homogeneous.

It is not hard to see that the hypothesis on the dimension of the local Killing algebras does imply quasihomogeneity of the metric (see Fact 4.1). For a quasihomogeneous Lorentz 3-manifold, the possible dimensions of the local Killing algebras $\mathfrak{k i l l}(x)$ are 3,4 or 6 (in the smooth case, this dimension may vary with the point $x$ ). Hence Theorem $\mathrm{B}$ deals with quasihomogeneous structures without open Kill ${ }^{\text {loc }}$-orbits having a 3 -dimensional local Killing algebra. 
Even if the conclusions of Theorem B are the same as for analytic metrics, the result can not be obtained by adapting the methods of $[\mathrm{DM}]$. Actually, we would like to point out that those regularity issues concentrate a great part of the subtilities in this kind of problems. To emphasize this aspect, we observe that the proof of Theorem $\mathrm{B}$ works for metrics of class $C^{9}$ (this regularity is required since we will need several covariant derivatives of the curvature tensor). This regularity is probably not optimal, but let us stress that the conclusions change dramatically if we work with metrics which have too low regularity.

TheOREM C. - There exist 3-dimensional Lorentz manifolds $\left(M^{3}, g\right)$, such that $g$ is $C^{1}$ and quasihomogeneous, satisfies hypotheses of Theorem B, but is not locally homogeneous. Moreover one can buildt compact examples in regularity $C^{0}$.

The conclusions of Theorem $\mathrm{C}$ will be made more precise in section 6 (see Theorems 6.1 and 6.2).

1.3. Organization of the paper. - A key ingredient in Gromov's theory of rigid transformation groups is a theorem about integration of finite order Killing fields. We will make a systematic use of this result in all our proofs, so that Section 2 will be devoted to a presentation of this theorem, in the convenient framework of Cartan geometries (which includes of course the case of Lorentz metrics). Next, we will use this integration result in Section 3, to prove two general criteria allowing to show that some quasihomogeneous pseudoRiemannian structures are actually locally homogeneous. Section 4 begins with a general study of local Lorentz actions of 4-dimensional Lie algebras on 3manifolds. This study, together with the criteria established in Section 3 lead to a proof of Theorem B. In Section 5, we explain how Theorem A can be deduced from Theorem B. The upshot is to show that when the isometry group of a pseudo-Riemannian manifold is topologically transitive, then numerous local Killing fields must appear (even if the isometry group is discrete, for instance). Finally, Section 6 will be devoted to the construction of examples of Theorem C.

\section{Integration of finite order Killing fields}

The main tool to understand the Kill ${ }^{\text {loc }}$-orbits of a rigid geometric structure is a theorem about integration of finite order Killing fields proved in [Gr] [Section 1.6]. The results of $[\mathrm{Gr}]$ generalize former integrability theorems proved by $\mathrm{K}$. Nomizu in [No] and I. Singer in [Si]. We won't follow here the approach of [Gr], but rather that of $[\mathrm{M}]$ and mostly $[\mathrm{P}]$. Those two papers present a general integrability result for Cartan geometries that will be the key ingredient in most of our proofs. We summarize below the results of [P] (first obtained in the analytical setting in $[\mathrm{M}]$, with a different approach). 
Let us begin with $(M, g)$, a pseudo-Riemannian manifold of type $(p, q)$. Let $\pi: \hat{M} \rightarrow M$ denote the bundle of orthonormal frames on $\hat{M}$. This is a principal $\mathrm{O}(p, q)$-bundle over $M$, and it is classical (see [KN][Chap. IV.2 ]) that the Levi-Civita connection associated to $g$ can be interpreted as an Ehresmann connection $\alpha$ on $\hat{M}$, with values in the Lie algebra $\mathfrak{o}(p, q)$. Let $\theta$ be the soldering form on $\hat{M}$, namely the $\mathbb{R}^{n}$-valued 1 -form on $\hat{M}$, which to every $\xi \in T_{\hat{x}} \hat{M}$ associates the coordinates of the vector $\pi_{*}(\xi) \in T_{x} M$ in the frame $\hat{x}$. The sum $\alpha+\theta$ is a 1 -form $\omega: T \hat{M} \rightarrow \mathfrak{o}(p, q) \ltimes \mathbb{R}^{n}$ called the canonical Cartan connection associated to $(M, g)$.

Pseudo-Riemannian structures of type $(p, q)$ are thus Cartan geometries modelled on the flat, type- $(p, q)$ Minkowski space $\mathbb{R}^{p, q}=\mathrm{O}(p, q) \ltimes \mathbb{R}^{n} / \mathrm{O}(p, q)$. The setting of Cartan geometries being really convenient for the kind of problems we are interested in, we give here the general definition.

A Cartan geometry $(M, \mathcal{C})$ modelled on a homogeneous space $X=G / P$ is the data of a triple $(M, \hat{M}, \omega)$ where $M$ is a manifold, $\pi: \hat{M} \rightarrow M$ is a $P$-principal bundle over $M$ and $\omega$, the Cartan connection, is a 1-form on $\hat{M}$ with values in the Lie algebra $\mathfrak{g}$. There are moreover extra properties satisfied by $\omega$.

- First, for every $\hat{x} \in \hat{M}, \omega_{\hat{x}}: T_{\hat{x}} \hat{M} \rightarrow \mathfrak{g}$ is an isomorphism of vector spaces.

- Moreover, the form $\omega$ is $P$-equivariant (where $P$ acts on $\mathfrak{g}$ via the adjoint action).

Beside pseudo-Riemannian metrics (which, as we just saw, correspond to $G=\mathrm{O}(p, q) \ltimes \mathbb{R}^{n}, n=p+q$, and $P=\mathrm{O}(p, q)$ when the type of the metric is $(p, q))$, quite a lot of other interesting geometric structures (linear connections, projective structures, conformal structures of dimension $\geq 3$ etc....) fit into this framework (see [CS, Chap. 4] for an extensive discussion of examples). The reader wanting to learn more about Cartan geometries will find modern and very comprehensive introductions in [CS] or [Sh].

2.1. Generalized curvature map. - We assume now that the structures considered are of class $C^{\infty}$. The curvature of the Cartan connection $\omega$ is a 2-form $K$ on $\hat{M}$, with values in $\mathfrak{g}$. If $X$ and $Y$ are two vector fields on $\hat{M}$, it is given by the relation:

$$
K(X, Y)=d \omega(X, Y)+[\omega(X), \omega(Y)] .
$$

Because at each point $\hat{x}$ of $\hat{M}$, the Cartan connection $\omega$ establishes an isomorphism between $T_{\hat{x}} \hat{M}$ and $\mathfrak{g}$, it follows that any $k$-differential form on $\hat{M}$, with values in some vector space $\mathcal{W}$, can be seen as a map from $\hat{M}$ to $\operatorname{Hom}\left(\otimes^{k} \mathfrak{g}, \mathcal{W}\right)$. This remark applies in particular for the curvature form, and we get a curvature map $\kappa: \hat{M} \rightarrow \mathcal{W}_{0}$, where the vector space $\mathcal{W}_{0}$ is $\operatorname{Hom}\left(\wedge^{2}(\mathfrak{g} / \mathfrak{p}) ; \mathfrak{g}\right)$ (the curvature is antisymmetric and vanishes when one argument is tangent to the fibers of $\hat{M})$. 
We can now differentiate $\kappa$, getting a map $D \kappa: T \hat{M} \rightarrow \mathcal{W}_{0}$. Our previous remark allows to see $D \kappa$ as a map $D \kappa: \hat{M} \rightarrow \mathcal{W}_{1}$, with $\mathcal{W}_{1}=\operatorname{Hom}\left(\mathfrak{g}, \mathcal{W}_{0}\right)$. Applying this procedure $r$ times, we define inductively the $r$-derivative of the curvature $D^{r} \kappa: \hat{M} \rightarrow \operatorname{Hom}\left(\otimes^{r} \mathfrak{g}, \mathcal{W}_{r}\right.$ ) (with $\mathcal{W}_{r}$ defined inductively by $\mathcal{W}_{r}=$ $\left.\operatorname{Hom}\left(\mathfrak{g}, \mathcal{W}_{r-1}\right)\right)$.

Let us now set $m=\operatorname{dim} G$. The generalized curvature map of the Cartan geometry $(M, \mathcal{C})$ is the map $\mathcal{D} \kappa=\left(\kappa, D \kappa, \ldots, D^{m} \kappa\right)$. The $P$-module $\mathcal{W}_{m}$ will be rather denoted $\mathcal{W}_{\mathcal{D} \kappa}$ in the following.

REMARK 2.1. - Note that in the case of pseudo-Riemannian structures, the generalized curvature map encodes the first $m+1$ covariant derivatives of the Riemann curvature tensor $R$.

2.2. Integrating finite order Killing vectors. - There is a natural notion of local isometry of a Cartan geometry $(M, \mathcal{C})$, as a local diffeomorphism $f: U \rightarrow V$ between open sets $U$ and $V$ of $M$, which can be lifted to a local diffeomorphism of $\hat{M}$ satisfying $f^{*} \omega=\omega$. In a same way, a local Killing field on some open subset $U \subset M$ is a vector field which can be lifted to a local vector field of $\hat{M}$ satisfying $L_{X} \omega=0$. Obviously, those notions coincide with the classical notion of local isometry, and local Killing field when our Cartan geometry is defined by a pseudo-Riemannian metric on a manifold.

For each integer $r \geq 1$, one defines $\operatorname{Ker}\left(D^{r} \kappa(\hat{x})\right)$ as the vector subspace of $\mathfrak{g}$ comprising all $\xi \in \mathfrak{g}$ such that $D^{r} \kappa(\hat{x})(\xi)=0$ (recall that $D^{r} \kappa(\hat{x})$ is a linear map from $\mathfrak{g}$ to $\left.\mathcal{W}_{r-1}\right)$.

It is clear that if $X$ is a local Killing field on $\hat{M}$ (namely $X$ satisfies $L_{X} \omega=0$ ), then all the maps $D^{r} \kappa$ are constant along the orbits the local flow $\varphi_{X}^{t}$. Thus $\omega(X(\hat{x})) \in \operatorname{Ker}\left(D^{j} \kappa(\hat{x})\right)$ for every $j \geq 1$, and every $\hat{x}$ where $X$ is defined. This leads naturally to a punctual notion of a Killing generator of order $r$ at $\hat{x}$, as a vector $\xi \in \mathfrak{g}$, such that $\xi$ belongs to $\bigcap_{j=1}^{r} \operatorname{Ker}\left(D^{j} \kappa(\hat{x})\right)$. We call $\operatorname{Kill}^{r}(\hat{x})$ the vector subspace of Killing generators of order $r$ at $\hat{x}$. If $m=\operatorname{dim} G$, we will note $\operatorname{Kill}^{\mathcal{D} \kappa}(\hat{x})$ instead of $\mathrm{Kill}^{m+1}(\hat{x})$.

A natural question is now: When is a Killing generator of order $r$ at $\hat{x}$ the evaluation of an actual local Killing field around $\hat{x}$ ?

2.2.1. The integrability theorem. - Motivated by the previous question, one defines the integrability locus of $\hat{M}$, denoted $\hat{M}^{\text {int }}$, as follows. A point $\hat{x} \in \hat{M}$ belongs to $\hat{M}^{\text {int }}$ if for every $\xi \in \operatorname{Kill}^{\mathcal{D} \kappa}(\hat{x})$, there exists a Killing field $X$ defined in a neighborhood of $\hat{x}$, and such that $\omega(X(\hat{x}))=\xi$. It is easily checked that $\hat{M}^{\text {int }}$ is a $P$-invariant set, and the integrability locus of $M$, denoted $M^{\text {int }}$, is just the projection of $\hat{M}^{\text {int }}$ on $M$. Since the dimension of $\mathrm{Kill}^{\mathcal{D} \kappa}(x)$ can only decrease locally, and because for every Killing field $X$, the vector $\omega(X(\hat{x}))$ belongs to $\operatorname{Kill}^{\mathcal{D} \kappa}(\hat{x})$, one gets that $M^{\text {int }}$ is an open subset of $M$. 
At first glance, the integrability locus $\hat{M}$ might be empty. It turns out that it is actually dense. It seems that the first result of this kind appears in [No, Th. 12], for Killing generators of infinite order (namely belonging to $\mathrm{Kill}^{r}$ for all $r \geq 1$ ). This result was greatly generalized by M. Gromov in [Gr]. One crucial improvement in Gromov's approach is that it is enough to consider only Killing generators of finite order (order which is moreover controlled by the dimension of $M$ and by the nature of the geometric structure). The precise statement we will need in this article is the following (compare to [Gr, Corollary 1.6.C], [M, Theorem 3.11], [P, Theorem 2]):

ThEOREM 2.2 (Integrability theorem). - Let $(M, \mathcal{C})$ be a smooth Cartan geometry. The integrability locus $M^{\text {int }}$ coincides with the subset of $M$ where the rank of the map $\mathcal{D} \kappa$ is locally constant. In particular, $M^{\text {int }}$ is a dense open subset of $M$.

Observe that because $\mathcal{D} \kappa$ is a $P$-equivariant map, the rank of $\mathcal{D} \kappa$ is constant along the fibers of $\pi: \hat{M} \rightarrow M$. Hence, it makes sense to speak about the rank of $\mathcal{D} \kappa$ at a point $x \in M$. More generally, we will allow in the following the notation $\mathcal{D} \kappa(x)$ for $x \in M$, meaning by this the $P$-orbit in $\mathcal{W}_{\mathcal{D} \kappa}$ of $\mathcal{D} \kappa(\hat{x})$, for $\hat{x}$ any point in the fiber of $x$.

Although it can be easily derived from the proofs given in $[\mathrm{P}]$, Theorem 2.2 does not appear with this precise statement. For the sake of completeness, we will explain at the end of this article (Section 7) how to deduce Theorem 2.2 from $[\mathrm{P}]$.

2.2.2. Components of the integrability locus and Kill ${ }^{\mathrm{oc}}$-algebra. - The dimension of the Lie algebra $\mathfrak{k i l l}^{\mathrm{loc}}(x)$ can not decrease locally, while the dimension of the vector space of $\operatorname{Kill}^{\mathcal{D} \kappa}(x)$ (the corank of $\mathcal{D} \kappa$ at $x$ ) can not increase locally. It follows from Theorem 2.2 that on $M^{\text {int }}$, the Lie algebra $\mathfrak{k i f l}^{\text {loc }}(x)$ is locally constant. Hence in restriction to $M^{\text {int }}$, $\mathfrak{k i l l}^{\text {loc }}(x)$ behaves as if the structure was analytic. More precisely $M^{\text {int }}$ splits into a union of connected components $\cup \mathcal{M}_{i}$, and on each $\mathcal{M}_{i}$ the Lie algebra $\mathfrak{k i l l}^{\text {loc }}(x)$ is the same for all $x \in \mathcal{M}_{i}$. We will denote it by $\mathfrak{k i r l}^{\text {loc }}\left(\mathcal{M}_{i}\right)$ in what follows.

For $x \in M$, we consider $\mathfrak{I}_{x}$, the isotropy algebra at $x$, namely the Lie algebra of local Killing fields defined in a neighborhood of $x$ and vanishing at $x$.

FACT 2.3. - If $x \in M^{\text {int }}$, then the isotropy algebra $\mathfrak{I}_{x}$ is isomorphic to the Lie algebra of the stabilizer of $\mathcal{D} \kappa(x)$ in $P$.

Proof: Let us consider $\hat{x} \in \hat{M}$ in the fiber of $x$. Every local Killing field $X$ around $x$ which vanishes at $x$, lifts to a local Killing field around $\hat{x}$, still denoted $X$, which is vertical at $\hat{x}$. We call $e v_{\hat{x}}$ the map $X \mapsto \omega(X(\hat{x}))$. The relation $\varphi_{X}^{t} \cdot \hat{x}=\hat{x} \cdot e^{t e v_{\hat{x}}(X)}$, available for $t$ in a neighborhood of 0 , together with the invariance of $\mathcal{D} \kappa$ under Killing flows, shows that $e v_{\hat{x}}$ a linear embedding from 
$\mathfrak{I}_{x}$ to the Lie algebra of the stabilizer of $\mathcal{D} \kappa(x)$ in $P$ (this map is one-to-one because a local Killing field on $\hat{M}$ vanishing at a point must be identically zero). Cartan's formula $L_{X}=\iota_{X} \circ d+d \circ \iota_{X}$ shows that whenever $X$ and $Y$ are two Killing fields around $\hat{x}$, the relation $\omega([X, Y])=K(X, Y)-[\omega(X), \omega(Y)]$ holds. When $X$ or $Y$ is vertical, $K(X, Y)=0$, proving that $e v_{\hat{x}}$ is an anti-morphism of Lie algebras. To see that $e v_{\hat{x}}$ is onto, let us consider $\left\{e^{t \xi}\right\}_{t \in \mathbb{R}}$, a 1-parameter group of $P$ fixing $\mathcal{D} \kappa(\hat{x})$. Clearly, $\xi$ belongs to $\operatorname{Kill}^{\mathcal{D} \kappa}(\hat{x})$, so that by Theorem $2.2, \omega^{-1}(\xi)$ is the evaluation at $\hat{x}$ of a local Killing field. This Killing field being vertical at $\hat{x}$, it corresponds to a local Killing field of $\mathfrak{I}_{x}$. $\diamond$

Theorem 2.2 ensures that if $x \in M^{\text {int }}$, $\operatorname{dim} \mathfrak{k i l l}^{\text {loc }}(x)$ coincides with the corank of $\mathcal{D} \kappa$ at $x$. Let us call $\mathcal{O}_{x}^{\mathcal{D} \kappa}$ the $P$-orbit of $\mathcal{D} \kappa(\hat{x})$ (it does not depend on the choice of $\hat{x}$ in the fiber of $x$, hence the notation). By Fact $2.3, \operatorname{dim} \mathcal{O}_{x}^{\mathcal{D} \kappa}=$ $\operatorname{dim} P-\operatorname{dim} \mathfrak{I}_{x}$. We conclude that for every $x \in M^{\text {int }}$ :

$$
\operatorname{dim} \mathfrak{k i l l}^{\text {loc }}(x)-\operatorname{dim} \mathfrak{I}_{x}=\operatorname{dim} M+\operatorname{dim} \mathcal{O}_{x}^{\mathcal{D} \kappa}-\operatorname{rk}(\mathcal{D} \kappa)(x) .
$$

Observe that if the geometry is locally homogeneous in a neighborhood of $x$ then $\operatorname{dim} \mathfrak{k i r l}^{\text {loc }}(x)-\operatorname{dim} \mathfrak{I}_{x}=\operatorname{dim} M$ and we have the equality $\operatorname{dim} \mathcal{O}_{x}^{\mathcal{D} \kappa}=$ $\operatorname{rk}(\mathcal{D} \kappa)(x)$.

\section{From quasihomogeneity to local homogeneity}

Using Theorem 2.2, we are going to single out some general situations where quasihomogeneity implies local homogeneity. We give below two criteria, which will be further implemented to prove Theorem B.

3.1. A first criterion for general Cartan geometries. - The first criterion we give is very easy, and applies to any Cartan geometry. That's why we state it with this generality. We recall from the previous section that any Cartan geometry $(M, \mathcal{C})$ modelled on some space $X=G / P$ admits a $P$-equivariant generalized curvature map $\mathcal{D} \kappa: \hat{M} \rightarrow \mathcal{W}_{\mathcal{D} \kappa}$, where $\mathcal{W}_{\mathcal{D} \kappa}$ is some vector space bearing a linear $P$-action.

We use in the following the notations introduced in Section 2.

Proposition 3.1. - Let $(M, \mathcal{C})$ be a connected Cartan geometry of algebraic type modelled on $X=G / P$, and $\mathcal{D} \kappa$ its generalized curvature map. Let $x_{0} \in M$ be a point satisfying the three following properties:

1. The Killoc -orbit of $x_{0}$ is open (in particular $x_{0} \in M^{\mathrm{int}}$ ).

2. The dimension of $\mathfrak{k i t l}^{\mathrm{loc}}\left(x_{0}\right)$ is minimal among all the dimensions of $\mathfrak{k i l l}^{\text {loc }}(y)$, when $y$ ranges over $M^{\text {int }}$.

3. The P-orbit of $\mathcal{D} \kappa\left(x_{0}\right)$ is closed in $\mathcal{W}_{\mathcal{D} \kappa}$.

Then $(M, \mathcal{C})$ is locally homogeneous. 
For every $\hat{x} \in \hat{M}$, let us denote by $\mathcal{O}_{\hat{x}}^{\mathcal{D} \kappa}$ the orbit of $\mathcal{D} \kappa(\hat{x})$ in $\mathcal{W}_{\mathcal{D} \kappa}$ (this orbit is the same for all the points in a same fiber, so we will also use the notation $\left.\mathcal{O}_{x}^{\mathcal{D} \kappa}\right)$. Recall from Section 2.2.2 that for every $x \in M^{\text {int }}$, we have the equality :

$$
\operatorname{dim} \mathfrak{k i l l l}^{\mathrm{loc}}(x)-\operatorname{dim} \mathfrak{I}_{x}=\operatorname{dim} M+\operatorname{dim} \mathcal{O}_{x}^{\mathcal{D} \kappa}-\operatorname{rk}(\mathcal{D} \kappa)(x) .
$$

The second hypothesis of the proposition says that $\operatorname{rk}(\mathcal{D} \kappa)(y) \leq \operatorname{rk}(\mathcal{D} \kappa)\left(x_{0}\right)$ for every $y \in M^{\text {int }}$, and hence for every $y \in M$ by density of $M^{\text {int }}$. We thus will write in the following $r_{\max }$ instead of $\operatorname{rk}(\mathcal{D} \kappa)\left(x_{0}\right)$. Equation (1), together with the first hypothesis of the proposition says that $\operatorname{dim} \mathcal{O}_{x_{0}}^{\mathcal{D} \kappa}=r_{\max }$.

Proposition 3.1 will be proved if we show that the Kill ${ }^{\text {loc }}$-orbit $\mathcal{O}_{x_{0}}^{\text {loc }}$, which is open by assumption, is also closed. To do this, let us pick $x$ in the boundary of $\mathcal{O}_{x_{0}}^{\text {loc }}$. The $P$-orbit $\mathcal{O}_{x}^{\mathcal{D} \kappa}$ is then in the closure of $\mathcal{O}_{x_{0}}^{\mathcal{D} \kappa}$, hence coincides with $\mathcal{O}_{x_{0}}^{\mathcal{D} \kappa}$ by the third hypothesis. By $P$-equivariance of $\mathcal{D} \kappa$, the $\operatorname{rank} \operatorname{rk}(\mathcal{D} \kappa)(x)$ is always at least the dimension of $\mathcal{O}_{x}^{\mathcal{D} \kappa}$. This yields the inequality $\operatorname{rk}(\mathcal{D} \kappa)(x) \geq r_{\max }$, which must actually be an equality by definition of $r_{\max }$. We infer that the rank of $\mathcal{D} \kappa$ is locally constant around $x$, implying $x \in M^{\text {int }}$ by Theorem 2.2. Equality (1) thus holds at $x$, leading to

$$
\operatorname{dim} \mathfrak{k i l l}^{\mathrm{loc}}(x)-\operatorname{dim} \mathfrak{I}_{x}=\operatorname{dim} M+\operatorname{dim} \mathcal{O}_{x_{0}}^{\mathcal{D} \kappa}-r_{\text {max }}=\operatorname{dim} \mathcal{O}_{x_{0}}^{\text {loc }}=\operatorname{dim} M .
$$

This shows that $\mathcal{O}_{x}^{\text {loc }}$ is open, hence meets $\mathcal{O}_{x_{0}}^{\text {loc }}$, and $x \in \mathcal{O}_{x_{0}}^{\text {loc }}$ as desired.

3.2. Homogeneous geodesic segments, and a second criterion. - We are now going to prove a second criterion, allowing to deduce local homogeneity of a pseudo-Riemannian structure.

Before stating the criterion, we must recall the definition of a homogeneous geodesic segment. If $(M, g)$ is a pseudo-Riemannian manifold, a geodesic segment $\gamma:(-\delta, \delta) \rightarrow M$ is said to be homogeneous whenever it coincides locally with a piece of (local) orbit of some local Killing field. For our purpose, let us make the following trivial remark: if some point of a homogeneous geodesic segment is contained in an open $\mathrm{Kill}^{\mathrm{loc}}$-orbit $\mathcal{O}$, then all this geodesic segment is contained in $\mathcal{O}$. Thus, if some locally homogeneous component of a pseudo-Riemannian manifold is modelled on a space admitting a lot of homogeneous geodesic segments, one can expect to saturate the component by "broken geodesic segments", in order to prove that the component is actually the whole manifold $M$.

We now make this remark into something more quantitative. For every $x \in M$ and $u \in T_{x} M$, denote by $\gamma_{u}$ the geodesic segment with initial datas $\gamma_{u}(0)=x$ and $\gamma_{u}^{\prime}(0)=u$. Let us introduce the set

$$
\mathcal{A}(u)=\left\{\lambda \in \mathbf{R} \mid \exists X \in \mathfrak{k i l l}^{\mathrm{loc}}(x), X(x)=u \text { and } \nabla_{u} X(x)=\lambda u\right\},
$$

and put

$$
\eta(u)=\inf \{|\lambda| \mid \lambda \in \mathcal{A}(u)\}
$$


We adopt the convention $\eta(u)=\infty$ when $\mathcal{A}(u)=\emptyset$. It is readily checked that $\gamma_{u}$ is homogeneous in a neighborhood of $x$ if and only if $\mathcal{A}(u) \neq \emptyset$. The key fact is that if $x$ belongs to the integrability locus $M^{\text {int }}$, we can estimate the "length" of this homogeneous geodesic segment inside $\gamma_{u}$ thanks to $\eta(u)$. More precisely:

Proposition 3.2. - Let $(M, g)$ be a pseudo-Riemannian manifold. Let $x_{0} \in$ $M^{\mathrm{int}}, u \in T_{x_{0}} M$, and $\delta>0$ such that the geodesic $\gamma_{u}$ is defined on the interval $[-\delta, \delta]$. Assume that $\mathcal{A}(u) \neq \emptyset$, and that the dimension of $\mathfrak{k i l l}^{\mathrm{loc}}\left(x_{0}\right)$ is minimal among all the dimensions of $\mathfrak{k i l l}^{\mathrm{loc}}(y)$, when $y$ ranges over $M^{\mathrm{int}}$. Then, if $\tau=$ $\min \left(\delta, \frac{1}{|\eta(u)|}\right)$, the geodesic segment $\gamma_{u}([-\tau, \tau])$ is homogeneous. In particular, if $\eta(u)=0, \gamma_{u}([-\delta, \delta])$ is homogeneous.

From proposition 3.2, we can derive the following criterion of local homogeneity.

Corollary 3.3. - Let $(M, g)$ be a connected pseudo-Riemannian manifold, and $T \mathcal{L} M$ be the bundle of lightlike tangent vectors. Assume that there exists $x_{0} \in M$ such that

1. The Kill ${ }^{\mathrm{loc}}$-orbit of $x_{0}$ is open.

2. The dimension of $\mathfrak{k i l l}^{\text {loc }}\left(x_{0}\right)$ is minimal among all the dimensions of $\mathfrak{k i l l}^{\text {loc }}(z)$, when $z$ ranges over $M^{\text {int }}$

3. For every compact subset $K \subset T \mathcal{L} M$, there exists $M_{K}>0$ such that $\eta(u) \leq M_{K}$ for every $u \in K \cap T \mathcal{O}_{x_{0}}^{\text {loc }}$.

Then $M$ coincides with the Killoc ${ }_{-}$orbit of $x_{0}$, hence is locally homogeneous.

The proof of Proposition 3.2 will be done in the next section. For the moment, let us explain how we derive Corollary 3.3 from the proposition.

We want to prove that every $y \in M$ belongs to the Kill ${ }^{\text {loc }}$-orbit $\mathcal{O}_{x_{0}}^{\text {loc }}$. Let us endow $M$, with an auxiliary Riemannian metric. The norm of a tangent vector $u$ for this metric will be denoted by $|u|$. Let us consider a path $\gamma$ joining $x_{0}$ to $y$, and a connected open set $S \subset M$ containing $\gamma$. We can choose $S$ with compact closure $\bar{S}$ in $M$. The set

$$
K=\left\{v \in T_{x} M|x \in \bar{S}, g(v, v)=0,| v \mid=1\right\}
$$

is a compact subset of $T \mathcal{L} M$. Let $M_{K}$ be the constant given by the third assumption in Corollary 3.3.

LEMMA 3.4. - It is possible to join $x_{0}$ and $y$ by a path made of broken lightlike geodesic segments $\gamma_{u_{i}} \subset S$, with $i=0, \ldots, s, u_{i} \in K$ and $\gamma_{u_{i}}$ defined on $\left[0, \epsilon_{i}\right]$, $0<\epsilon_{i}<\frac{1}{M_{K}}$

Proof: Let $x \in S$ be any point, and $U \subset S$ a neighborhood of $x$. If $U$ is chosen small enough, there exists on $U$ a frame field $\left(E_{1}, \ldots, E_{n}\right)$ such that 
$g\left(E_{i}, E_{i}\right)=0$ and $\left|E_{i}\right|=1$ for $i=1, \ldots, n$. In what follows, we adopt the notation $\varphi_{i}^{s}(z)$ instead of $\exp \left(z, s E_{i}(z)\right)$. There exists $0<\epsilon<\frac{1}{M_{K}}$ small enough, so that the smooth map $\psi:\left(t_{1}, \ldots, t_{n}\right) \mapsto \varphi_{n}^{t_{n}}\left(\varphi_{n-1}^{t_{n-1}}\left(\ldots\left(\varphi_{1}^{t_{1}}(x)\right)\right)\right.$ makes sense on $(-\epsilon, \epsilon)^{n}$. Because $\frac{\partial \psi}{\partial t_{i}}(0)=E_{i}(x)$, the inverse mapping theorem ensures that $\psi$ yields a diffeomorphism from some $\left(-\epsilon^{\prime}, \epsilon^{\prime}\right)^{n}$ with $0<\epsilon^{\prime}<\epsilon$, to an open neighborhood $W$ of $x$ in $S$. In other words, points of $W$ can be joined to $x$ by a path made of broken lightlike geodesic segments.

The previous argument shows that the set of points in $S$ which can be joined to $x_{0}$ by a path made of broken lightlike geodesic segments as in the statement of Lemma 3.4 is nonempty and open. But it also shows that the complement of this set is open, hence empty since $S$ is connected. This proves the lemma. $\diamond$

We can now apply repeatedly Proposition 3.2 to get that all the segments $\gamma_{u_{i}}$ given by Lemma 3.4 are homogeneous, hence $y$ belongs to $\mathcal{O}_{x_{0}}^{\text {loc }}$.

3.3. Proof of Proposition 3.2. - In what follows, we use the notations of Section 3.2. The set $\mathcal{A}(u)$, which is assumed to be nonempty, is an affine subspace of $\mathbf{R}$, hence it is either $\mathbf{R}$ or a point. One infers easily the existence of $X \in \mathfrak{k i f l}^{\mathrm{loc}}(x)$ such that $X\left(x_{0}\right)=u$ and $\nabla_{u} X\left(x_{0}\right)=\eta(u) X\left(x_{0}\right)$. We consider $\hat{x}_{0} \in \hat{M}$ in the fiber of $x_{0}$, and we lift $X$ to a neighborhood $\hat{U}$ of $\hat{x}_{0}$ in $\hat{M}$, obtaining a field (still denoted $X$ ) which satisfies $L_{X} \omega=0$. For every $\hat{y} \in \hat{U}$, we put $\xi(\hat{y})=\omega(X(\hat{y}))$.

3.3.1. Exponential flow in $\hat{M}$. - The Cartan connection $\omega$ on $\hat{M}$ yields the notion of $\omega$-constant vector field on $\hat{M}$. If $v \in \mathfrak{g}$ and $\hat{x} \in \hat{M}$, we can consider the local flow $\varphi_{v}^{t}$ of the $\omega$-constant vector field with value $v$, around $\hat{x}$. When this flow is defined up to time 1 , we define $\exp (\hat{x}, v)$ as $\varphi_{v}^{1}$. The exponential map at $\hat{x}: v \mapsto \exp (\hat{x}, v)$, is thus defined on a neighborhood of $0 \in \mathfrak{g}$.

Observe that the projections of curves $t \mapsto \exp (\hat{x}, t v)$ on $M$ are geodesics for the metric $g$ when $v$ is horizontal, namely $v \in \mathbb{R}^{n} \subset \mathfrak{o}(p, q) \ltimes \mathbb{R}^{n}$. For arbitrary $v$, we get by projection a wider class of curves (for instance in the flat case, we get all orbits under 1-parameter groups of $\left.\mathrm{O}(p, q) \ltimes \mathbb{R}^{n}\right)$.

3.3.2. Covariant derivative seen in $\hat{M}$. - The covariant derivative $\nabla_{u} X\left(x_{0}\right)$ can be interpreted in the following way in $\hat{M}$ (see for instance $[\mathrm{KN}][$ chap. III, $\S 1$ and $\S 2])$. There is a unique vector $v \in \mathbb{R}^{n} \subset \mathfrak{o}(p, q) \ltimes \mathbb{R}^{n}$ satisfying $\pi_{*}\left(\omega_{\hat{x}_{0}}^{-1}(v)\right)=u$. Now, the horizontal component of $(v \cdot \xi)\left(\hat{x}_{0}\right)$ (namely the component on $\left.\mathbb{R}^{n} \subset \mathfrak{o}(p, q) \ltimes \mathbb{R}^{n}\right)$ is again identified by $\pi_{*} \circ \omega_{\hat{x}_{0}}^{-1}$ with a vector of $T_{x_{0}} M$ which is precisely $\nabla_{u} X\left(x_{0}\right)$.

Let us now decompose $\xi=\xi\left(\hat{x}_{0}\right)$ into a sum $v+A$, where $v \in \mathbf{R}^{n}$ and $A \in \mathfrak{o}(p, q)$. In the following, we adopt the notation $\hat{\gamma}(t)=\exp \left(\hat{x}_{0}, t v\right)$, and 
$\xi(t)=\xi(\hat{\gamma}(t))$. Recall the formula for the curvature $K$ of $\omega$ :

$$
d \omega(X, V)+[\omega(X), \omega(V)]=K(X, V)
$$

where $V$ is any vector field on $\hat{U}$. Applying this formula when $V$ is the $\omega$ constant vector field such that $\omega(V)=v$, and using the fact that the Killing vector field $X$ commutes with $V$ (because $L_{X} \omega=0$ ), one derives easily the following differential equation satisfied by $\xi(t)$ :

$$
\xi^{\prime}(t)=[\xi(t), v]-\kappa(\hat{\gamma}(t))(\xi(t), v)
$$

Because the curvature $K$ takes its values in $\mathfrak{o}(p, q)$ (this is because LeviCivita's connection is torsion-free), the horizontal component of $\xi^{\prime}(0)$ is $[A, v]$. Thus our hypothesis $\nabla_{u} X\left(x_{0}\right)=\eta(u) X\left(x_{0}\right)$ is equivalent to $[A, v]=\eta(u) v$.

3.3.3. Saturation by the exponential flow. - We are now going to prove an important invariance property of the component of $x_{0}$ with respect to the exponential flow.

LEMmA 3.5. - Let us consider $I=(\alpha, \beta)$ a maximal interval of definition of $\left.\hat{\gamma}: t \mapsto \exp \left(\hat{x}_{0}, t \xi\right)\right)$. Then the differential of the map $\mathcal{D} \kappa$ is constant on $\hat{\gamma}(I)$. In particular, the projection of $\hat{\gamma}(I)$ on $M$ is homogeneous.

Proof: We denote by $D(\mathcal{D} \kappa)$ the differential of the map $\mathcal{D} \kappa: \hat{M} \rightarrow \mathcal{W}_{\mathcal{D} \kappa}$. As already observed in section 2 , it can be seen as a map from $\hat{M}$ to $\operatorname{Hom}\left(\mathfrak{g}, \mathcal{W}_{\mathcal{D} \kappa}\right)$. Let us consider $\Lambda=\left\{t \in I \mid D(\mathcal{D} \kappa)(\hat{\gamma}(t))=D(\mathcal{D} \kappa)\left(\hat{x}_{0}\right)\right\}$. This is clearly a closed set of $I$. It is also nonempty because for $t$ in a small interval around 0 , $\exp (\hat{x}, t \xi)$ is the orbit of a Killing field, so that $D(\mathcal{D} \kappa)(\hat{\gamma}(t))$ is constant on this interval. Now if $t_{0} \in \Lambda, D(\mathcal{D} \kappa)\left(\hat{\gamma}\left(t_{0}\right)\right)=D(\mathcal{D} \kappa)\left(\hat{x}_{0}\right)$. Recall that for any $\hat{x} \in$ $\hat{M}, \operatorname{Ker}(D(\mathcal{D} \kappa)(\hat{x}))$ denotes the set of vectors $v \in \mathfrak{g}$ satisfying $D(\mathcal{D} \kappa)(\hat{x})(v)=$ $0_{\mathcal{W}}$. Observe also that the dimension of $\operatorname{Ker}(D(\mathcal{D} \kappa)(\hat{x}))$ coincides with the corank of $\mathcal{D} \kappa$ at $\hat{x}$. We thus obtain that the rank of $\mathcal{D} \kappa$ is the same at $\hat{\gamma}\left(t_{0}\right)$ and at $\hat{x}_{0}$. The second hypothesis of Proposition 3.2 says that this rank is maximal, hence locally constant. Since $\xi$ is in the Kernel of $D(\mathcal{D} \kappa)\left(\hat{x}_{0}\right)$, hence in the kernel of $D(\mathcal{D} \kappa)\left(\hat{\gamma}\left(y_{0}\right)\right)$, we can apply Theorem 2.2: there exists a local Killing field $Y$ around $\hat{\gamma}\left(t_{0}\right)$ such that $\omega\left(Y\left(\hat{\gamma}\left(t_{0}\right)\right)\right)=\xi$. It follows that $D(\mathcal{D} \kappa)$ is constant on some $\hat{\gamma}\left(\left[t_{0}-\epsilon, t_{0}+\epsilon\right)\right)$ for $\epsilon>0$. The set $\Lambda$ is thus open, and we are done.

The arguments above show that for every $t_{0} \in I$, there is a small segment $\hat{\gamma}(] t_{0}-\epsilon, t_{0}+\epsilon[)$ which is the orbit of $\hat{\gamma}\left(t_{0}\right)$ under the local flow of a local Killing field $Y$. Projecting on $M$, we get that the segment $\pi(\hat{\gamma}(I))$ is homogeneous. $\diamond$

3.3.4. End of the proof of Proposition 3.2. - We now make the link between the projection of $\hat{\gamma}(I)$ on $M$ and the segment $\gamma_{u}$ thanks to the following reparametrization lemma. 
Lemma 3.6 (Reparametrization lemma). - Let $\xi \in \mathfrak{g}$. Assume that $\xi=A+$ $v$, with $A \in \mathfrak{o}(p, q)$ and $v \in \mathbf{R}^{n}$. Assume moreover that $[A, v]=\eta v$. Then the following equality holds for every $t$ such that at least one member makes sense:

1. If $\eta=0, \exp (\hat{x}, t \xi)=\exp (\hat{x}, t v) \cdot e^{t A}$.

2. If $\eta \neq 0, \exp (\hat{x}, t \xi)=\exp \left(\hat{x}, \frac{1}{\eta}\left(e^{\eta t}-1\right) v\right) \cdot e^{t A}$.

Proof: Let us make a first observation : two curves $t \mapsto \alpha(t)$ and $t \mapsto \beta(t)$ in $\hat{M}$ satisfy an identity of the form $\alpha(t)=\beta(t) . p(t)$, for some curve $t \mapsto p(t)$ with values in $\mathrm{O}(p, q)$ if and only if

$$
\omega\left(\alpha^{\prime}(t)\right)=A d(p(t))^{-1} \omega\left(\beta^{\prime}(t)\right)+\omega_{G}\left(p^{\prime}(t)\right),
$$

where $\omega_{G}$ stands for the Maurer-Cartan form on the Lie group $G=\mathrm{O}(p, q) \ltimes$ $\mathbf{R}^{n}$. As a consequence, we have a relation of the form

$$
\exp (\hat{x}, t \xi)=\exp (\hat{x}, f(t) v) \cdot p(t)
$$

for some function $f$, if and only if

$$
\xi=f^{\prime}(t) A d(p(t))^{-1} \cdot v+\omega_{G}\left(p^{\prime}(t)\right),
$$

which in turns is equivalent to the identity

$$
e^{t \xi}=e^{f(t) v} p(t)
$$

in the Lie group $G$. It is thus enough to check the reparametrization formulas in $G$.

For $m \geq 1$, we have

$$
\left(\begin{array}{cc}
t A & t v \\
0 & 0
\end{array}\right)^{m}=\left(\begin{array}{cc}
t^{m} A^{m} & t^{m} \eta^{m-1} v \\
0 & 0
\end{array}\right)
$$

Hence

$$
e^{t \xi}=\left(\begin{array}{c}
e^{t A}\left(t+\frac{t^{2} \eta}{2 !}+\frac{t^{3} \eta^{2}}{3 !}+\ldots\right) v \\
0
\end{array}\right)
$$

This shows that $e^{t \xi}=e^{t v} \cdot e^{t A}$ if $\eta=0$, and $e^{t \xi}=e^{\frac{1}{\eta}\left(e^{\eta t}-1\right) v} \cdot e^{t A}$ if $\eta \neq 0$, as desired. $\diamond$

We now get easily Proposition 3.2, observing that the geodesic $\gamma_{u}$ is the projection on $M$ of the curve $t \mapsto \exp \left(\hat{x}_{0}, t v\right)$. If we are in the first case of the reparametrization lemma (namely the case $\eta(u)=0$ ), then we get directly that $\pi(\hat{\gamma}(I))$ contains $\gamma_{u}([-\tau, \tau])$ (here $\left.\tau=\delta\right)$. By the previous remarks, $\gamma_{u}([-\tau, \tau])$ is then homogeneous. If we are the second case of Lemma 3.6, it is better to rewrite the reparametrization relation in the following way :

$$
\exp (\hat{x}, s v)=\exp \left(\hat{x}, \frac{1}{\eta(u)} \ln (\eta(u) s+1)\right) e^{-\frac{1}{\eta(u)} \ln (\eta(u) s+1) A},
$$

expression which makes sense as soon as $|s| \leq \tau$ where $\tau=\min \left\{\delta ; \frac{1}{|\eta(u)|}\right\}$. Again, $\pi(\hat{\gamma}(I))$ contains $\gamma_{u}([-\tau, \tau])$ and we are done. 


\section{Proof of Theorem B}

In this section, we are under the asumptions of Theorem B. The manifold $(M, g)$ is 3-dimensional and Lorentzian, and on a dense open subset, the dimension of the Lie algebra of local Killing fields is at least 4-dimensional.

4.1. Hyperbolic, elliptic and parabolic components. - By the general discussion made in Section 2.2.2, the integrability locus $M^{\text {int }}$ is a disjoint union of connected components: $M^{\text {int }}=\bigcup \mathcal{M}_{i}$. On each component $\mathcal{M}$, there is a well-defined Lie algebra $\mathfrak{k i l l}^{\text {loc }}(\mathcal{M})$ of local Killing fields, which by our assumption is at least 4 dimensional. Hence, for every $x \in \mathcal{M}$, the stabilizers of the $\mathrm{O}(1,2)$-orbit $\mathcal{D} \kappa(x)$ are always 1 or 3 -dimensional, the points where they are 3 dimensional being those at which the sectional curvature is constant. Because in a finite dimensional linear representation of $\mathrm{O}(1,2)$, no point can have a stabilizer of dimension exactly 2, and because of Fact 2.3, the possible dimension for $\mathfrak{k i f l}^{\mathrm{loc}}(\mathcal{M})$ is 4 or 6 . In the latter case, the sectional curvature is constant on $\mathcal{M}$.

Assume now that the component $\mathcal{M}$ does not have constant sectional curvature. The dimension of $\mathfrak{k i r l}^{\mathrm{loc}}(\mathcal{M})$ is then 4 , and we can still formulate the

FACT $4.1([\mathrm{DM}])$. — The component $\mathcal{M}$ is locally homogeneous.

The proof of [DM, Lemma 4] can be applied directly here. Either all $x \in \mathcal{M}$ have 1-dimensional isotropy algebra and we are done. Or there exists some point $x \in \mathcal{M}$ such that $\mathfrak{I}_{x}$ is of dimension 3 . The isotropy representation is then the 3-dimensional irreducible representation of $\mathfrak{s o}(1,2)$, and the 1-dimensional Kill ${ }^{\text {loc }}$-orbit of $x$ yields an invariant line for this representation : contradiction.

If $x \in \mathcal{M}$, the local flow defined by a generator of $\mathfrak{I}_{x}$ determines a 1 parameter flow $\varphi^{t}$ of $\mathrm{SO}\left(T_{x} M\right)$, hence of $\mathrm{SO}(1,2)$. Up to conjugacy, there are three kinds of flows in $\mathrm{SO}(1,2)$ : hyperbolic, elliptic or parabolic (unipotent). We call the point $x$ hyperbolic, elliptic or parabolic, according to the type of $\varphi^{t}$. The component $\mathcal{M}$ itself will be called hyperbolic (resp. elliptic, resp. parabolic) if it contains an hyperbolic (resp. elliptic, resp. parabolic) point. This terminology makes sense, because $\mathcal{M}$ being locally homogeneous, the isotropy is of the same kind at each point.

4.2. Hyperbolic or elliptic components imply local homogeneity. Let us prove Theorem B when there exists a component $\mathcal{M} \subset M^{\text {int }}$ which is hyperbolic (resp. elliptic). Let us pick $x_{0} \in \mathcal{M}$ which is hyperbolic or elliptic. We are now in position to apply our first homogeneity criterion, given by Proposition 3.1. The two first assumptions of Proposition 3.1 are fulfilled by $x_{0}$ (under the hypothesis of Theorem B). To check that the third condition is also satisfied, we remark that in a finite dimensional representation of $\mathrm{O}(1,2)$, an orbit is closed if and only if the corresponding orbit of $\operatorname{SO}^{\circ}(1,2)$ is closed. 
Now, $\operatorname{SO}^{\circ}(1,2)$ is isomorphic to $\operatorname{PSL}(2, \mathbb{R})$, so that the third condition follows from the next lemma.

LEMmA 4.2. - Let $\rho: \operatorname{PSL}(2, \mathbb{R}) \rightarrow \mathrm{GL}(V)$ be a finite dimensional representation of $\operatorname{PSL}(2, \mathbb{R})$. Let $v \in V$ be a vector the stabilizer of which is an hyperbolic or elliptic 1-parameter group of $\operatorname{PSL}(2, \mathbb{R})$. Then the $\operatorname{PSL}(2, \mathbb{R})$-orbit of $v$ is closed in $V$.

This fact is certainly standard. For the sake of completeness, we will provide a proof at the end of the paper (see Section 8).

4.3. The geometry of parabolic components. - The previous section reduces the proof of Theorem $\mathrm{B}$ to the case when all the components of the integrability locus either have constant sectional curvature, or are parabolic. The general strategy to prove Theorem B will be to understand in more details the homogeneous spaces on which parabolic components are modelled, then to observe that those components have quite a lot of homogeneous geodesic segments, and finally to show that Corollary 3.3 can be applied.

The main result we have in mind in this section is the description of the possible Kill ${ }^{\text {loc }}$ algebras for a parabolic component.

Proposition 4.3. - Let $\mathcal{M}$ be a component of the integrability locus $M^{\text {int }}$ of a 3-dimensional Lorentz manifold $(M, g)$. We assume that $\mathcal{M}$ is parabolic. Then:

1. If the scalar curvature of $\mathcal{M}$ is 0 , the Lie algebra $\mathfrak{k i r l}^{\mathrm{loc}}(\mathcal{M})$ is isomorphic to $\mathbb{R} \ltimes_{h} \mathfrak{h e i s}, \mathbb{R} \ltimes_{e} \mathfrak{h e i s}$ or $\mathbb{R} \ltimes_{\nu} \mathfrak{h e i s}$, with $\nu \in \mathbb{R}^{*}$.

2. If the scalar curvature is nonzero on $\mathcal{M}$, then $\mathfrak{k i n l}^{\text {loc }}(\mathcal{M})$ is isomorphic to $\mathfrak{s l}(2, \mathbb{R}) \oplus \mathbb{R}$.

The notations used in this proposition will be explained in Section 4.3 .1 below. Proposition 4.3 yields a partial description of 4-dimensional Lie algebras admitting a local action on a 3-dimensional Lorentz manifolds, which is a question of independent interest. The full classification can be carried out with the same methods, eventhough we won't present it here.

4.3.1. Some extensions of heis by $\mathbb{R}$. - Let us make some algebraic preliminaries about 4-dimensional Lie algebras which are a semidirect product $\mathbb{R} \ltimes \mathfrak{h e i s}$, where $\mathfrak{h}$ eis stands for the 3-dimensional Heisenberg Lie algebra.

Let us consider a Lie algebra $\mathfrak{g} \simeq \mathbb{R} \ltimes \mathfrak{h e i s}$ with basis $X, Y, Z, T$, where $X, Y, Z$ generates the Lie subalgebra $\mathfrak{h e i s}$, with relation $[X, Y]=Z$ (and all other brackets involving $X, Y, Z$ zero), and $\operatorname{ad}(T)$ is given in the basis $X, Y, Z$ by a matrix

$$
\left(\begin{array}{cc}
A & 0 \\
0 & \operatorname{Tr}(A)
\end{array}\right) .
$$


If we replace $T$ by $\alpha T, \alpha \neq 0$, we won't affect the isomorphism type of our Lie algebra. The same is true if for some $P \in \mathrm{GL}(2, \mathbb{R})$, we replace $X, Y$ by $P . X, P . Y$, and $Z$ by $\operatorname{det}(P) . Z$. Thus, we can replace $A$ by any multiple $\alpha A$, $\alpha \neq 0$, or a conjugate $P A P^{-1}$ with $P \in \mathrm{GL}(2, \mathbb{R})$. We end up with the following few cases:

1. Unimodular case. If the trace of $A$ is 0 , we may choose

$$
A=0, A=\left(\begin{array}{cc}
1 & 0 \\
0 & -1
\end{array}\right), A=\left(\begin{array}{ll}
0 & 1 \\
0 & 0
\end{array}\right) \text { or } A=\left(\begin{array}{cc}
0 & 1 \\
-1 & 0
\end{array}\right) \text {. }
$$

The corresponding Lie algebras are denoted respectively :

$\mathbb{R} \times \mathfrak{h e i s}, \mathbb{R} \ltimes_{h} \mathfrak{h e i s}, \mathbb{R} \ltimes_{p} \mathfrak{h e i s}$ and $\mathbb{R} \ltimes_{e} \mathfrak{h e i s . ~}$

2. Scalar case. If $A$ is a nonzero scalar matrix, we may choose $A=\left(\begin{array}{ll}1 & 0 \\ 0 & 1\end{array}\right)$, and we denote the corresponding Lie algebra by $\mathbb{R} \ltimes_{s} \mathfrak{h} \mathfrak{i s}$.

3. Other nonunimodular cases. In all other cases, the matrix $A$ is not scalar and has a nonzero trace, that we might assume to be 2 . This leads to three subcases
(a) Hyperbolic case: $A=\left(\begin{array}{cc}1+\lambda & 0 \\ 0 & 1-\lambda\end{array}\right), \lambda>0$.
(b) Unipotent case: $A=\left(\begin{array}{ll}1 & 1 \\ 0 & 1\end{array}\right)$.
(c) Elliptic case: $A=\left(\begin{array}{cc}1 & \frac{1}{\lambda} \\ -\frac{1}{\lambda} & 1\end{array}\right), \lambda>0$.

Observe that in case (3) above (namely $A$ is not scalar and $\operatorname{Tr}(A) \neq 0$ ), the number $\nu=\frac{\operatorname{det}(A)}{\operatorname{Tr}(A)^{2}}$ is a complete invariant of isomorphism classes. We will then denote by $\mathbb{R} \ltimes_{\nu} \mathfrak{h} \mathfrak{e i s}$ this last family of algebras. The parameter $\nu$ can take any real value. For $\nu<1 / 4$, we are in the hyperbolic case above, the parabolic and elliptic cases corresponding to $\nu=1 / 4$ and $\nu>1 / 4$ respectively.

4.3.2. The curvature module. - We consider on $\mathbb{R}^{3}$ the Lorentzian form, with matrix in a basis $e, h, f$ given by:

$$
J=\left(\begin{array}{lll}
0 & 0 & 1 \\
0 & 1 & 0 \\
1 & 0 & 0
\end{array}\right)
$$

We call $\mathrm{O}(1,2)$ the subgroup of $\mathrm{GL}(3, \mathbb{R})$ preserving the bilinear form determined by $J$. Its Lie algebra is denoted by $\mathfrak{o}(1,2)$, and admits the following basis :

$$
E=\left(\begin{array}{ccc}
0 & 1 & 0 \\
0 & 0 & -1 \\
0 & 0 & 0
\end{array}\right), H=\left(\begin{array}{ccc}
1 & 0 & 0 \\
0 & 0 & 0 \\
0 & 0 & -1
\end{array}\right)
$$




$$
F=\left(\begin{array}{ccc}
0 & 0 & 0 \\
1 & 0 & 0 \\
0 & -1 & 0
\end{array}\right)
$$

We thus have the commutation relations $[H, E]=E,[H, F]=-F$ and $[E, F]=$ $H$.

Let $\left(M^{3}, g\right)$ be 3 -dimensional Lorentz manifold, and denote by $\hat{M}$ its bundle of orthonormal frames. At each $\hat{x} \in \hat{M}$, the curvature $\kappa(\hat{x})$ is an element of $\operatorname{Hom}\left(\wedge^{2}\left(\mathbb{R}^{3}\right), \mathfrak{o}(1,2)\right)$. Because of Bianchi's identities, the curvature module is actually a 6 -dimensional submodule of $\operatorname{Hom}\left(\wedge^{2}\left(\mathbb{R}^{3}\right), \mathfrak{o}(1,2)\right)$. Choosing $e \wedge h$, $e \wedge f, h \wedge f$ as a basis for $\wedge^{2}\left(\mathbb{R}^{3}\right)$, and $E, H, F$ as a basis for $\mathfrak{o}(1,2)$, an element of $\operatorname{Hom}\left(\wedge^{2}\left(\mathbb{R}^{3}\right), \mathfrak{o}(1,2)\right)$ is merely given by a $3 \times 3$ matrix. The action of $\mathrm{O}(1,2)$ on $\operatorname{Hom}\left(\wedge^{2}\left(\mathbb{R}^{3}\right), \mathfrak{o}(1,2)\right)$ corresponds to the conjugation on matrices.

Scalar matrices are $\mathrm{O}(1,2)$-invariant, and form a 1-dimensional irreducible submodule (corresponding to constant sectional curvature).

The other irreducible submodule of the curvature module is 5-dimensional, spanned by the matrices:

$$
\begin{gathered}
\left(\begin{array}{lll}
0 & 0 & 1 \\
0 & 0 & 0 \\
0 & 0 & 0
\end{array}\right),\left(\begin{array}{lll}
0 & 1 & 0 \\
0 & 0 & 1 \\
0 & 0 & 0
\end{array}\right),\left(\begin{array}{ccc}
1 & 0 & 0 \\
0 & -2 & 0 \\
0 & 0 & 1
\end{array}\right), \\
\left(\begin{array}{lll}
0 & 0 & 0 \\
1 & 0 & 0 \\
0 & 1 & 0
\end{array}\right),\left(\begin{array}{lll}
0 & 0 & 0 \\
0 & 0 & 0 \\
1 & 0 & 0
\end{array}\right) .
\end{gathered}
$$

We call $\kappa_{0}$ the element of $\operatorname{Hom}\left(\wedge^{2}\left(\mathbb{R}^{3}\right), \mathfrak{o}(1,2)\right)$ corresponding to the identity matrix, namely $\kappa_{0}$ maps $e \wedge h$ to $E, e \wedge f$ to $H$ and $h \wedge f$ to $F$. We also call $\kappa_{1}$ the element of $\operatorname{Hom}\left(\wedge^{2}\left(\mathbb{R}^{3}\right), \mathfrak{o}(1,2)\right)$ corresponding to the matrix $\left(\begin{array}{lll}0 & 0 & 1 \\ 0 & 0 & 0 \\ 0 & 0 & 0\end{array}\right)$.

The two dimensional vector space spanned by $\kappa_{0}$ and $\kappa_{1}$ is the set of fixed points of the action of $\left\{e^{t E}\right\}_{t \in \mathbb{R}}$ on the curvature module.

4.3.3. Equation of Killing fields. - Cartan's formula for the Lie derivative $L_{X}=\iota_{X} \circ d+d \circ \iota_{X}$ yields that whenever $U$ and $V$ are two local Killing fields on an open subset of $\hat{M}$, the following relation holds:

$$
\omega([U, V])=\kappa(\omega(U) \wedge \omega(V))-[\omega(U), \omega(V)])
$$

Let $\mathcal{M}$ be a parabolic component, and $x$ a point of $\mathcal{M}$ having an open Kill ${ }^{\text {loc }}$-orbit (see Fact 4.1). Because $\mathcal{M}$ is parabolic, there exists a local Killing field $Y$, vanishing at $x$, and generating a parabolic flow of $\mathrm{SO}(1,2)$ in $T_{x} M$. Thus, there exists $\hat{x}$ in the fiber of $x$ such that after lifting $Y$ into a Killing field around $\hat{x}$, we have $\omega_{\hat{x}}(Y(\hat{x}))=E$. We can also find 3 other local Killing fields $T, X, Z$ such that at $\hat{x}$ :

$$
\omega(Z)=e+\mu H+\zeta F,
$$




$$
\omega(X)=h+\alpha H+\theta F \text { and } \omega(T)=f+\beta H+\gamma F .
$$

Observe also that at $\hat{x}$, the curvature $\kappa(\hat{x})$ is invariant by the action of $\left\{e^{t E}\right\}$, hence is of the form $\sigma(x) \kappa_{0}+b(\hat{x}) \kappa_{1}$. We wrote $\sigma(x)$ instead of $\sigma(\hat{x})$ since this number does not depend on the point $\hat{x}$ in the fiber of $x$. Actually, $x \mapsto \sigma(x)$ coincides with (a constant multiple of) the scalar curvature. Since $\mathcal{M}$ is locally homogeneous (see Fact 4.1), $\sigma$ is constant on $\mathcal{M}$. To simplify the notations, we will write $b$ instead of $b(\hat{x})$ (keeping in mind, though, that it depends on $\hat{x}$ ). This yields the relations

$$
\kappa(e \wedge h)=\sigma E, \kappa(e \wedge f)=\sigma H, \kappa(h \wedge f)=b E+\sigma F .
$$

Relation (3) expressed for the 4 Killing fields $X, Y, Z, T$ provides 6 equations in $\mathfrak{o}(1,2) \ltimes \mathbb{R}^{3}$ involving $\alpha, \beta, \gamma, \mu, \theta, \zeta$, as well as the two auxiliary parameters $\sigma$ and $b$. We are going to solve those equations explicitely below.

4.3.4. Bracket relations and proof of proposition 4.3. - Let us introduce the vector space

$$
\mathcal{E}=\operatorname{Span}(\omega(X(\hat{x})), \omega(Y(\hat{x})), \omega(Z(\hat{x})), \omega(T(\hat{x}))) .
$$

Relations (5) yield the following identities at $\hat{x}$ :

$$
\begin{array}{lcccc}
\omega([T, Y])=-[\omega(T), \omega(Y)]= & -h-\beta E+\gamma H & =\omega(-X-\beta Y)+(\alpha+\gamma) H+\theta F, \\
\omega([Y, X])=-[\omega(Y), \omega(X)]= & -e+\alpha E & = & \omega(\alpha Y-Z)+\mu H, \\
\omega([Z, Y])=-[\omega(Z), \omega(Y)]=-[e+\mu H+\zeta F, E]= & -\mu \omega(Y)+\zeta H .
\end{array}
$$

Because all the expressions above must belong to $\mathcal{E}$, we infer

$$
\alpha=-\gamma \text { and } \theta=\zeta=\mu=0 .
$$

Moreover, since a local Killing field $U$ satisfying $\omega(U(\hat{x}))=0$ must be zero (a local flow of isometries must act freely on the bundle of orthonormal frames $\hat{M})$, we also get the identities

$$
[T, Y]=-X-\beta Y,[X, Y]=Z-\alpha Y \text { and }[Y, Z]=0 .
$$

Relations (6) allow to update (4), and we get at $\hat{x}$ the new identities :

$$
\omega(Z)=e, \omega(Y)=E \text { and } \omega(X)=h+\alpha H .
$$

We then write 3 more equations:

$$
\begin{aligned}
\omega([Z, X]) & =\kappa(e \wedge h)-[e, h+\alpha H]=\sigma E+\alpha e=\omega(\alpha Z+\sigma Y) \\
\omega([T, X]) & =\kappa(f \wedge h)+[h+\alpha H, f+\beta H-\alpha F] \\
& =-2 \alpha f-b E+\left(\alpha^{2}-\sigma\right) F \\
& =\omega(-2 \alpha T-b Y)+2 \alpha \beta H-\left(\alpha^{2}+\sigma\right) F \\
\omega([T, Z]) & =\kappa(f \wedge e)+[\omega(Z), \omega(T)]=-\sigma H+[e, f+\beta H-\alpha F] \\
& =\omega(\alpha X-\beta Z)-\left(\alpha^{2}+\sigma\right) H .
\end{aligned}
$$


which imply, by the same arguments as above

$$
\alpha \beta=0 \text { and } \alpha^{2}=-\sigma .
$$

as well as 3 more bracket relations:

(9) $[X, Z]=-\alpha Z-\sigma Y,[T, X]=-2 \alpha T-b Y$, and $[T, Z]=\alpha X-\beta Z$.

This leads to two cases according to the value of the scalar curvature $\sigma$.

Case of zero scalar curvature. - Because of relation (8), the hypothesis $\sigma=0$ also implies $\alpha=0$. Relations (7) and (9) imply $[Y, Z]=[X, Z]=0,[X, Y]=Z$, so that the Lie algebra $\mathfrak{h}$ generated by $X, Y, Z$ is isomorphic to $\mathfrak{h e i s}$, and is an ideal in $\mathfrak{k i l l}^{\mathrm{loc}}(x)$. Moreover the adjoint action of $T$ on $\mathfrak{h}$, expressed in the basis $Y, X, Z$ is given by the matrix

$$
\operatorname{ad}(T)=\left(\begin{array}{ccc}
-\beta & -b & 0 \\
-1 & 0 & 0 \\
0 & 0 & -\beta
\end{array}\right) .
$$

The Lie algebra $\mathfrak{k i f l}^{\text {loc }}(x)$ is a semi-direct product $\mathbb{R} \ltimes \mathfrak{h e i s}$ described by the matrix $A=\left(\begin{array}{cc}-\beta & -b \\ -1 & 0\end{array}\right)$, as explained in Section 4.3.1. We see that the matrix $A$ is never scalar. Moreover, observe that while $b=b(\hat{x})$ has no precise meaning on $M$, its vanishing has one. It means that the sectional curvature is constant at $x$. In particular, because the Kill ${ }^{\text {loc }}$-orbit of $\hat{x}$ is open, and because $\mathcal{M}$ does not have constant sectional curvature (on any open set) by hypothesis, we see that $b \neq 0$. As a consequence, the matrix $A$ is invertible. Hence $\mathfrak{k i f l}^{\mathrm{loc}}(x)$ is isomorphic to the Lie algebra $\mathbb{R} \ltimes_{h} \mathfrak{h e i s}$ (case $\beta=0, b<0$ ), $\mathbb{R} \ltimes_{e} \mathfrak{h e i s}$ (case $\beta=0, b>0$ ), or to the Lie algebra $\mathbb{R} \ltimes_{\nu} \mathfrak{h e i s}$, where $\nu=\frac{-b}{\beta^{2}}$ (here $\beta \neq 0$ ) can take all nonzero real values.

Case of nonzero scalar curvature. - Relation (8) yields $\sigma=-\alpha^{2}$, so that $\sigma$ turns out to be negative. Also, (8) shows that $\beta=0$. The bracket relations become:

$$
\begin{gathered}
{[T, Z]=\alpha X \quad[T, Y]=-X \quad[T, X]=-2 \alpha T-b Y,} \\
{[X, Z]=-\alpha Z+\alpha^{2} Y \quad[X, Y]=Z-\alpha Y \quad[Z, Y]=0 .}
\end{gathered}
$$

Let us put $Z^{\prime}=\frac{-1}{4 \alpha^{2}}(Z+\alpha Y), Y^{\prime}=\frac{1}{4 \alpha^{2}}(Z-\alpha Y), X^{\prime}=\frac{-1}{2 \alpha} X$ and $T^{\prime}=$ $T-\frac{b}{2} Y^{\prime}-b Z^{\prime}$.

It is easily checked that now, $Z^{\prime}$ lies in the center of $\mathfrak{k i l l}^{\text {loc }}(x)$. Moreover:

$$
\left[X^{\prime}, Y^{\prime}\right]=\frac{-1}{8 \alpha^{3}}[X, Z-\alpha Y]=\frac{2 \alpha}{8 \alpha^{3}}(Z-\alpha Y)=Y^{\prime}
$$




$$
\begin{aligned}
{\left[X^{\prime}, T^{\prime}\right] } & =\left[X^{\prime}, T\right]-\frac{b}{2}\left[X^{\prime}, Y^{\prime}\right]=-T-\frac{b}{2 \alpha} Y-\frac{b}{2} Y^{\prime} \\
& =-T+\frac{b}{2} Y^{\prime}+b Z^{\prime} \\
& =-T^{\prime}
\end{aligned}
$$

and $\left[Y^{\prime}, T^{\prime}\right]=\left[Y^{\prime}, T\right]=-\frac{1}{2 \alpha} X=X^{\prime}$.

Thus, the Lie algebra $\mathfrak{k i l l}^{\text {loc }}(x)$ is isomorphic to a product $\mathfrak{s l}(2, \mathbb{R}) \oplus \mathbb{R}$.

4.4. End of the proof of Theorem B. - By Section 4.2, we can assume that all the components of $M^{\text {int }}$ are parabolic or of constant sectional curvature. If all are of constant sectional curvature, then $(M, g)$ itself has constant sectional curvature (by density of $\left.M^{\text {int }}\right)$, and $(M, g)$ is locally homogeneous. We thus assume that there exists at least one parabolic component $\mathcal{M}$, and we want to apply Corollary 3.3 in order to show that $M$ is locally homogeneous. Any point $x_{0} \in \mathcal{M}$ having an open orbit satisfies the two first conditions of Corollary 3.3. The harder part is to check the third condition.

Let us consider the closed subset $\hat{F} \subset \hat{M}$ defined as $\hat{F}=\kappa^{-1}\left(\operatorname{Span}\left(\kappa_{0}, \kappa_{1}\right)\right)$. Because we excluded the presence of hyperbolic and elliptic components, we know that in each fiber of $\hat{M}$, there is a point where $\kappa$ is fixed by the 1-parameter group $\left\{e^{t E}\right\}_{t \in \mathbb{R}}$. It follows that $\hat{F}$ projects onto $M$. By the very definition of $\hat{F}$, there exist two continuous functions $\sigma$ and $b$ on $\hat{F}$, such that for every $\hat{x} \in \hat{F}$, $\kappa(\hat{x})=\sigma(\hat{x}) \kappa_{0}+b(\hat{x}) \kappa_{1}$. Actually, because $\sigma$ is constant along the fibers, and because $M$ is locally homogeneous on a dense open set, the function $\sigma$ is locally constant on a dense open subset of $\hat{M}$, hence constant. We will thus write $\sigma$ instead of $\sigma(\hat{x})$.

At each $\hat{x} \in \hat{M}$, there is a linear map $\iota_{\hat{x}}: \mathfrak{g} \rightarrow T_{x} M$ defined by $\iota_{\hat{x}}(u)=$ $\pi_{*}\left(\omega_{\hat{x}}^{-1}(u)\right)$. This map $\iota_{\hat{x}}$ is an isomorphism between $\mathbb{R}^{3} \subset \mathfrak{o}(1,2) \ltimes \mathbb{R}^{3}$ and $T_{x} M$.

In the statement below, we will denote by $f_{t}$ the vector $A d\left(e^{t E}\right) . f$.

LEMMA 4.4. - Let $\mathcal{M}$ be a parabolic component, $x$ a point of $\mathcal{M}$, and $\hat{x}$ a point of $\hat{F}$ projecting on $x$. Then

1. If $\mathfrak{g}$ is isomorphic to $\mathfrak{s l}(2, \mathbb{R}) \oplus \mathbb{R}, \mathbb{R} \ltimes_{h} \mathfrak{h e i s}$ or $\mathbb{R} \ltimes_{e} \mathfrak{h e i s}$, then $\eta\left(\iota_{\hat{x}}(e)\right)=$ $0=\eta\left(\iota_{\hat{x}}\left(f_{t}\right)\right)$ for all $t \in \mathbb{R}$.

2. If $\mathfrak{g}$ is isomorphic to $\mathbb{R} \ltimes_{\nu} \mathfrak{h e i s , ~} \nu \in \mathbb{R}^{*}$, then $\eta\left(\iota_{\hat{x}}(e)\right)=0$ and for all $t \in \mathbb{R}, \eta\left(\iota_{\hat{x}}\left(f_{t}\right)\right) \leq \sqrt{\frac{|b(\hat{x})|}{|\nu|}}$.

Proof: If we look at the proof of Proposition 4.3 given in Section 4.3.4, we see that in each case (zero or nonzero scalar curvature), and for each $\hat{x} \in \hat{F}$, we have three Killing fields $X, Y, T$ in $\mathfrak{k i l l}^{\text {loc }}$ satisfying:

1. If $\mathfrak{g}$ is isomorphic to $\mathfrak{s l}(2, \mathbb{R}) \oplus \mathbb{R}, \mathbb{R} \ltimes_{h} \mathfrak{h} \mathfrak{e i s}$ or $\mathbb{R} \ltimes_{e} \mathfrak{h e i s}, \omega(X)=e, \omega(Y)=$ $E, \omega(T)=f-\alpha F$, with $\alpha \in \mathbb{R}$ (evaluation made at $\hat{x}$ ). 
2. If $\mathfrak{g}$ is isomorphic to $\mathbb{R} \ltimes_{\nu} \mathfrak{h e i s}, \nu \in \mathbb{R}^{*}, \omega(X)=e, \omega(Y)=E, \omega(T)=$ $f+\beta H$, with $\beta \neq 0$ satisfying $\beta^{2}=\frac{-b(\hat{x})}{\nu}$ (again, the evaluation is made at $\hat{x})$.

This leads immediately to $\nabla_{\iota_{\hat{x}}(e)} X(x)=0$. Because $F(f)=0$ and $H(f)=$ $-f$, one has $\nabla_{\iota_{\hat{x}}(f)} T(x)=0$ in the first case and $\nabla_{\iota_{\hat{x}}(f)} T(x)=-\beta T(x)$ in the second one, where $\beta= \pm \sqrt{\frac{|b(\hat{x})|}{\nu}}$. Both cases lead to the conclusion of the lemma for $t=0$.

Now the local flow of $Y$, denoted $Y^{t}$, acts on $\mathfrak{k i l l l o c}(x)$. For every $t \in \mathbb{R}, Y^{t}$ is defined on a small neighborhood of $x$, and $T_{t}=\left(Y^{t}\right)^{*} T$ is a local Killing field around $x$. One checks that $T_{t}(x)=\iota_{\hat{x}}\left(f_{t}\right)$. Because the local flow $Y^{t}$ preserves $\nabla$ one still has $\nabla_{\iota_{\hat{x}}\left(f_{t}\right)} T_{t}(x)=0$ in the first case above and $\nabla_{\iota_{\hat{x}}\left(f_{t}\right)} T_{t}(x)=$ $-\beta T_{t}(x)$ in the second one, which establishes the lemma. $\diamond$

We consider $K$ a compact subset of $T \mathcal{L} M$, projecting on a compact set $C \subset M$, and such that $C \cap \mathcal{M} \neq \emptyset$. Let us take a compact subset $\hat{C} \subset \hat{M}$ which project on $C$. We can saturate $\hat{C}$ by the action of $\mathrm{O}(2) \subset \mathrm{O}(1,2)$, and we denote again $\hat{C}$ the compact set obtained in this way. Observe that for every $\hat{x} \in \hat{M}$, the orbit of $\hat{x}$ under the right action of $\mathrm{O}(2)$ meets $\hat{F}$. This is just because the conjugates of $\mathbb{R} . E$ under $\mathrm{O}(2)$ describe all the parabolic directions in $\mathfrak{s o}(1,2)$ (i.e directions generating a parabolic 1-parameter group). Thus, we can replace $\hat{C}$ by $\hat{F} \cap \hat{C}$, obtaining a compact subset which still projects onto $C$.

Let us now endow $\mathbb{R}^{3}$ with a norm |.|. By compactness of $\hat{C}$ and $K$, there exist positive $C_{1}$ and $C_{2}$ such that $\iota_{\hat{x}}^{-1}(u) \leq\left|C_{1}\right|$ and $|b(\hat{x})| \leq C_{2}$ for every $\hat{x} \in \hat{C}$ and $u \in K$.

It is clear that $\lim _{|t| \rightarrow \infty}\left|A d\left(e^{t E}\right) \cdot f\right|=\infty$, so that there exists $C_{3}>0$ with $\min \left\{|e|, \inf _{t \in \mathbf{R}}\left|A d\left(e^{t E}\right) . f\right|\right\} \geq C_{3}$.

Let us pick a lightlike vector $u \in K \cap T \mathcal{M}$. Let us choose any $\hat{x}$ in $\hat{C}$ that projects on $x$. We thus have either $\iota_{\hat{x}}^{-1}(u)= \pm\left|\iota_{\hat{x}}^{-1}(u)\right| e$, or $\iota_{\hat{x}}(u)=$ $\pm \frac{\left|\iota_{\hat{x}}^{-1}(u)\right|}{\left|f_{t}\right|} f_{t}$ for some $t \in \mathbb{R}$. In the first case, Lemma 4.4 says that $\eta(u)=0$. In the second case, we use first the fact that $\eta$ is 1-homogeneous to get $\eta(u)=$ $\frac{\left|\iota_{\hat{x}}^{-1}(u)\right|}{\left|f_{t}\right|} \eta\left(\iota_{\hat{x}}\left(f_{t}\right)\right)$. Then we apply Lemma 4.4 and obtain

$$
\eta(u) \leq \frac{C_{1} \sqrt{C_{2}}}{\sqrt{|\nu|} C_{3}}
$$

The third condition of Corollary 3.3 is satisfied, and the proof of Theorem $\mathrm{B}$ is complete. 


\section{Dense orbit implies locally homogeneous}

Let us now explain how one can deduce Theorem A from Theorem B. We start with a smooth, closed 3-dimensional Lorentz manifold $\left(M^{3}, g\right)$, and our assumption is that $\operatorname{Iso}(M, g)$ admits a dense orbit. Then Gromov's theorem 1.1 ensures that the integrability locus $M^{\text {int }}$ is quasihomogeneous. Actually, because there is a dense orbit for $\operatorname{Iso}(M, g)$, all the components of $M^{\text {int }}$ are pairwise isometric. Thus the Lie algebra $\mathfrak{k i f l}^{\mathrm{loc}}(\mathcal{M})$ does not depend on the component $\mathcal{M}$, and will be merely denoted $\mathfrak{k i r l}{ }^{\text {loc }}$. Since we are in a quasihomogeneous situation, the dimension of this algebra is 3, 4 or 6 (we already noticed that 5 is not allowed). If $\mathfrak{k i l l}^{\text {loc }}$ is 6 -dimensional, then $g$ has constant sectional curvature on $M^{\text {int }}$, hence on $M$ by density of $M^{\text {int }}$ in $M$, and $(M, g)$ is indeed locally homogeneous. If $\mathfrak{k i l l}^{\mathrm{loc}}$ is 4-dimensional, then Theorem B applies, and we conclude again that $(M, g)$ is locally homogeneous. Hence, Theorem A will be proved if we can show that $\mathfrak{g}$ is not 3 -dimensional. This is at this point that we will use the compactness of $M$ (actually a finite volume assumption would be enough).

Observe that we can assume $\operatorname{Iso}(M, g)$ noncompact, otherwise $(M, g)$ would be directly homogeneous under the action of $\operatorname{Iso}(M, g)$. Observe also that whenever $\mathfrak{k i f l}^{\text {loc }}$ is 3 -dimensional, the isotropy algebra $\mathfrak{I}_{x}$ must be trivial for every $x \in M^{\text {int }}$. The conclusion will thus be a direct consequence of the following general observation:

Proposition 5.1. - Let $(M, g)$ be a compact pseudo-riemannian manifold. If the group Iso $(M, g)$ is noncompact, then for almost every $x \in M^{\mathrm{int}}$, the isotropy algebra $\mathfrak{I}_{x}$ generates a noncompact subgroup of $\mathrm{O}\left(T_{x} M\right)$

Proof: We denote by $(p, q)$ the signature of $g, \hat{M}$ the bundle of orthonormal frames on $M$, and $\mathcal{D} \kappa$ the generalized curvature map. Recall from section 2.1 that $\mathcal{D} \kappa$ has range into the $\mathrm{O}(p, q)$ module $\mathcal{W}_{\mathcal{D} \kappa}=\operatorname{Hom}\left(\otimes^{m+1} \mathfrak{g}, \mathcal{V}\right)$, where $\mathcal{V}$ is $\operatorname{Hom}\left(\wedge^{2}\left(\mathbb{R}^{n}\right) ; \mathfrak{o}(p, q) \ltimes \mathbb{R}^{n}\right)$, and $m=\frac{(p+q)(p+q+1)}{2}$.

Let us first recall the following recurrence theorem :

LEmma 5.2 (Poincaré recurrence, see [FK], Theorem 2.2.6)

Let $G$ be a Lie group acting continuously on a manifold $M$, and preserving a finite Borel measure $\nu$. Then for almost every $x \in M$, there exists a sequence $\left(g_{k}\right)$ leaving every compact subset of $G$, and a sequence $\left(x_{k}\right)$ converging to $x$, so that $g_{k} . x_{k}$ converges to $x$.

The lemma applies to $(M, g)$ because a closed Lorentz manifold naturally defines a finite Borel measure which is invariant by isometries (the density giving volume 1 to every orthonormal frame). The set of points in $M^{\text {int }}$ which are recurrent for Iso $(M, g)$ has thus full measure in $M^{\text {int }}$. Let $x$ be such a point, and $\hat{x} \in \hat{N}$ in the fiber of $x$. The recurrence hypothesis means that there exists 
$\left(f_{k}\right)$ tending to infinity in $\operatorname{Iso}(M, g)$, and $\left(p_{k}\right)$ a sequence of $\mathrm{O}(p, q)$ such that $f_{k}(\hat{x}) \cdot p_{k}^{-1}$ tends to $\hat{x}$. Observe that $\left(p_{k}\right)$ tends to infinity in $\mathrm{O}(p, q)$, because Iso $(M, g)$ acts properly on $\hat{M}$. By equivariance of $\mathcal{D} \kappa$, we also have

$$
p_{k} \cdot \mathcal{D} \kappa(\hat{x}) \rightarrow \mathcal{D} \kappa(\hat{x}) .
$$

The action of the algebraic group $\mathrm{O}(p, q)$ on $\mathcal{W}_{\mathcal{D} \kappa}$ is linear, hence algebraic. As a consequence, all the orbits of $\mathrm{O}(p, q)$ are locally closed. It is in particular the case of the orbit $\mathcal{O}$ of $\mathcal{D} \kappa(\hat{x})$. If $I$ denotes the stabilizer of $\mathcal{D} \kappa(\hat{x})$ in $\mathrm{O}(p, q)$, then the orbital map $\mathrm{O}(p, q) / I \rightarrow \mathcal{O}$ is an homeomorphism, when $\mathcal{O}$ is endowed with the topology induced by that of $\mathcal{W}_{\mathcal{D} \kappa}$. As a consequence, the property $p_{k} \cdot \mathcal{D} \kappa(\hat{x}) \rightarrow \mathcal{D} \kappa(\hat{x})$ implies the existence of a sequence $\left(\epsilon_{k}\right)$ in $\mathrm{O}(p, q)$ with $\epsilon_{k} \rightarrow I d$ and $\epsilon_{k} \cdot p_{k} \cdot \mathcal{D} \kappa(\hat{x})=\mathcal{D} \kappa(\hat{x})$. Since $\left(p_{k}\right)$ tends to infiny, so does $\left(\epsilon_{k} \cdot p_{k}\right)$, proving that $I$ is noncompact. Because $I$ is an algebraic group, the identity component $I^{o}$ is noncompact, and we conclude thanks to Fact 2.3.

$\diamond$

\section{Constructing quasihomogeneous metrics of lower regularity}

Theorem B is stated in the framework of smooth lorentz structures. Actually, a closer look at the proof shows that we need a regularity of the metric yielding a $C^{1}$ generalized curvature map $\mathcal{D} \kappa$. Hence, because the generalized curvature map involves, for 3-dimensional Lorentz metrics, the 6 first covariant derivatives of the curvature, our proof is actually available for metrics of class $C^{9} \ldots$.

It is likely that Theorem B holds for metrics of lower regularity. However, we are going to exhibit $C^{1}$ quasihomogeneous Lorentz metrics which are not locally homogeneous. Hence, regularity at least $C^{2}$ is necessary to get Theorem B. Here is our statement:

Theorem 6.1. - There exist $C^{1}$ lorentzian 3-manifolds $(M, g)$ which are quasihomogeneous but not locally homogeneous. More precisely, for any pair $\left(\nu_{1}, \nu_{2}\right)$ of distinct numbers in $\left(-\infty, \frac{1}{4}\right)$, one can buildt a $C^{1}$ Lorentz manifold $(M, g)$ having the following properties:

1. There exists an open subset $U_{1} \subset M$ which is locally homogeneous, with local Killing algebra isomorphic to $\mathbb{R} \ltimes_{\nu_{1}} \mathfrak{h}$ eis.

2. There exists an open subset $U_{2} \subset M$ which is locally homogeneous, with local Killing algebra isomorphic to $\mathbb{R} \ltimes_{\nu_{2}}$ heis.

3. The union $U_{1} \cup U_{2}$ is dense in $M$.

We refer to Section 4.3.1 for the notation $\mathbb{R} \ltimes_{\nu} \mathfrak{h e i s}$, and the classification of extensions of $\mathfrak{h e i s}$ by $\mathbb{R}$.

Actually, if we drop the regularity to $C^{0}$, it is even possible to get quasi homogeneous Lorentz metrics on compact manifolds. 
TheOREM 6.2. - There are $C^{0}$ Lorentz metrics on the 3-torus which are quasihomogeneous but not homogeneous.

The constructions work as follows. Let us endow $\mathbb{R}^{3}$ with coordinates $\left(x_{1}, x_{2}, x_{3}\right)$, and let $g_{0}=-2 d x_{1} d x_{3}+d x_{2}^{2}$ be the Minkowski metric. If $\alpha \in \mathbb{R}^{*}$, we can define on the open set $x_{3}>0$ the metric $g_{\alpha}=x_{3}^{\alpha} g_{0}$.

We introduce the 3 vector fields:

$$
X=x_{2} \frac{\partial}{\partial x_{1}}+x_{3} \frac{\partial}{\partial x_{2}}, \quad Y=\frac{\partial}{\partial x_{2}}, \quad Z=\frac{\partial}{\partial x_{1}} .
$$

Those are Killing fields for the flat metric $g_{0}$, and because they have vanishing component along $\frac{\partial}{\partial x_{3}}$, they are also Killing fields for $g_{\alpha}$.

There is a fourth vector field $T=2 \frac{\alpha+1}{\alpha} x_{1} \frac{\partial}{\partial x_{1}}+x_{2} \frac{\partial}{\partial x_{2}}-\frac{2}{\alpha} x_{3} \frac{\partial}{\partial x_{3}}$ which is also Killing for $g_{\alpha}$. Indeed, the flow $\varphi^{t}$ generated by $T$ is the linear flow

$$
e^{t}\left(\begin{array}{ccc}
e^{\frac{\alpha+2}{\alpha} t} & 0 & 0 \\
0 & 1 & 0 \\
0 & 0 & e^{-\frac{\alpha+2}{\alpha} t}
\end{array}\right),
$$

hence $\left(\varphi^{t}\right)^{*} g_{0}=e^{2 t} g_{0}$ and

$$
\left(\varphi^{t}\right)^{*} g_{\alpha}=\left(e^{\left(1-\frac{\alpha+2}{\alpha}\right) t} x_{3}\right)^{\alpha} e^{2 t} g_{0}=g_{\alpha} .
$$

The only nontrivial bracket relations between $T, X, Y, Z$ are $[X, Y]=Z$, $[T, X]=\frac{\alpha+2}{\alpha} X,[T, Y]=Y$ and $[T, Z]=2 \frac{\alpha+1}{\alpha} Z$

Hence the Lie algebra generated by $X, Y, Z$ is isomorphic to heis, and the Lie algebra $\mathfrak{g}$ generated by $T, X, Y, Z$ is an extension of $\mathfrak{h} \mathfrak{e} \mathfrak{s}$ by $\mathbb{R}$. Referring to the classification given in Section 4.3.1, we see that:

- If $\alpha=-1$, then $\mathfrak{g}$ is isomorphic to $\mathbb{R} \ltimes_{s} \mathfrak{h} \mathfrak{e i s}$.

- If $\alpha \neq-1, \mathfrak{g}$ is isomorphic to some algebra $\mathbb{R} \ltimes_{\nu} \mathfrak{h e i s . ~ A c t u a l l y ~ t h e ~}$ parameter $\nu$ is given by the formula $\nu=\frac{\operatorname{det}(A)}{\operatorname{Tr}(A)^{2}}$, where $A$ is the matrix $\left(\begin{array}{cc}\frac{\alpha+2}{\alpha} & 0 \\ 0 & 1\end{array}\right)$. Hence $\nu=\frac{1}{4}\left(1-\frac{1}{(\alpha+1)^{2}}\right)$. In particular, we see that the set of possible values for $\nu$ is exactly $\left(-\infty, \frac{1}{4}\right)$.

The local isometric action of the Lie algebra $\mathfrak{g}$ integrates into an action of a 4-dimensional Lie group $G$ (we can directly describe $G$ as a subgroup of affine transformations of $\mathbb{R}^{3}$ ) which is transitive on the set $x_{3}>0$. Hence the metric $g_{\alpha}$ are always homogeneous. Let us now explain why the Lie algebra $\mathfrak{g}$ coincides with the Lie algebra of local Killing fields of $g_{\alpha}$. If this latter Lie algebra were bigger than $\mathfrak{g}$, then it should be 6-dimensional (as already observed, there are no 3-dimensional Lorentz metrics with a 5-dimensional local Killing algebra), hence of constant curvature. It is however readily checked that $g_{\alpha}$ does not have constant curvature.

Let now $\nu_{1}$ and $\nu_{2}$ be as in the statement of Theorem 6.1. We choose $\alpha_{1}$ and $\alpha_{2}$ in $(-\infty,-1)$ such that $\nu_{1}=\frac{1}{4}\left(1-\frac{1}{\left(\alpha_{1}+1\right)^{2}}\right)$ and $\nu_{2}=\frac{1}{4}\left(1-\frac{1}{\left(\alpha_{2}+1\right)^{2}}\right)$. There 
will be a unique $z \in(1,+\infty)$ such that $\alpha_{2}=\alpha_{1} \frac{z}{z-1}$. Finally we introduce the metric $\tilde{g}_{\alpha_{2}}=z^{\alpha_{1}}(z-1)^{-\alpha_{2}}\left(x_{3}-1\right)^{\alpha_{2}} g_{0}$ (which is a smooth Lorentz metric for $\left.x_{3}>1\right)$.

On the open set $M$ given by $x_{3}>1$, we define a Lorentz metric $g$ as follows

- On the set $1<x_{3} \leq z, g=g_{\alpha_{1}}$.

- On the set $x_{3}>z, g=\tilde{g}_{\alpha_{2}}$.

We claim that $g$ has the properties of Theorem 6.1. It is smooth outside the set $x_{3}=z$. The metrics $g_{\alpha_{1}}$ and $\tilde{g}_{\alpha_{2}}$ coincide along $x_{3}=z$. Because of the condition $\alpha_{2}=\alpha_{1} \frac{z}{z-1}$, their first derivatives also coincide at $x_{3}=z$, so that the metric $g$ is $C^{1}$. On the open set $U_{1}$ defined by $1<x_{3}<z, g$ coincides with $g_{\alpha_{1}}$, hence its local Killing algebra is isomorphic to $\mathbb{R} \ltimes_{\nu_{1}} \mathfrak{h}$ eis. On the open set $U_{2}$, the local Killing algebra of $g$ is that of $\tilde{g}_{\alpha_{2}}$. But if $\psi$ denotes the translation $x \mapsto x-e_{3}, \tilde{g}_{\alpha_{2}}$ is nothing but $z^{\alpha_{1}}(z-1)^{-\alpha_{2}} \psi^{*} g_{\alpha_{2}}$, hence its local Killing algebra is isomorphic to that of $g_{\alpha_{2}}$, namely $\mathbb{R} \ltimes_{\nu_{2}} \mathfrak{h e i s . ~ T h i s ~ p r o v e s ~}$ Theorem 6.1.

To get $C^{0}$ quasihomogeneous metrics on the 3 -torus, we do as follows. We first choose $\alpha_{1} \in(-\infty,-1)$ and $\alpha_{2} \in(1,+\infty)$ such that $\nu_{1}=\frac{1}{4}\left(1-\frac{1}{\left(\alpha_{1}+1\right)^{2}}\right)$ and $\nu_{2}=\frac{1}{4}\left(1-\frac{1}{\left(\alpha_{2}+1\right)^{2}}\right)$ are not equal. The two metrics $g_{\alpha_{1}}$ and $g_{\alpha_{2}}$ coincide on the hyperplane $x_{3}=1$. Let us consider the strip $\bar{\Omega}$ given by $\frac{1}{2} \leq x_{3} \leq 2^{\alpha_{2}-\alpha_{1}}$, and define $g$ on $\bar{\Omega}$, by $g=g_{\alpha_{1}}$ if $\frac{1}{2} \leq x_{3} \leq 1$ and $g=g_{\alpha_{2}}$ on $1 \leq x_{3} \leq 2^{\alpha_{2}-\alpha_{1}}$. This yields a $C^{0}$ quasihomogeneous Lorentz metric on $\Omega$. Now, observe that $g$ is invariant by the translations of vectors $e_{1}$ and $e_{2}$, and that the translation of vector $\left(2^{\alpha_{2}-\alpha_{1}}-\frac{1}{2}\right) e_{3}$ yields an isometric identification of the restriction of $g$ to the hyperplanes $x_{3}=\frac{1}{2}$ and $x_{3}=2^{\alpha_{2}-\alpha_{1}}$. After those identifications, $g$ induces a $C^{0}$ quasihomogeneous metric on $\mathbb{T}^{3}$.

Observe that we could make more complicated examples, using a countable family of $g_{\alpha_{i}}$, and producing quasihomogenous metrics for which the locus of homogeneity has infinitely many connected components.

\section{Annex A: Integrability locus}

Let $(M, \hat{M}, \omega)$ be a Cartan geometry with model space $X=G / P$. We denote in the following by $m$ the dimension of $\mathfrak{g}$. If we fix $\left(e_{1}, \ldots, e_{m}\right)$ a basis of $\mathfrak{g}$, we get a parallelism $\mathcal{P}$ on $\hat{M}$ defined by the vector fields $X_{i}=\omega^{-1}\left(e_{i}\right), i=1 \ldots, m$. As explained in [P] [Sec. 4.3], it is enough to prove Theorem 2.2 for the Cartan geometry defined by $\mathcal{P}$ on $\hat{M}$ (which is a Cartan geometry modelled on the abelian Lie group $\mathbb{R}^{m}$ ). Indeed, if we work on $\hat{M}$, the Killing generators of order $r \geq 1$ are the same for both geometries, and the Killing fields are also the same. 
7.0.1. Curvature of the parallelism. - Let $\mathcal{P}$ be the parallelism on $\hat{M}$ as defined above. The curvature of $\mathcal{P}$ is the 2 -form on $\hat{M}$ defined by

$$
K\left(X_{i}, X_{j}\right)=-\omega\left(\left[X_{i}, X_{j}\right]\right) \text {, for every pair of integers } 1 \leq i \leq j \leq n .
$$

Let us write $\left[X_{i}, X_{j}\right]=\Sigma_{i, j} \gamma_{i j}^{k} X_{k}$, where the $\gamma_{i j}^{k}$ are functions on $\hat{M}$. At each $\hat{x} \in \hat{M}$, the curvature is seen as an element of $\operatorname{Hom}\left(\wedge^{2}(\mathfrak{g}), \mathfrak{g}\right)$ given by the formula $\kappa(\hat{x})\left(e_{i}, e_{j}\right)=\Sigma_{i, j} \gamma_{i j}^{k}(\hat{x}) e_{k}$. The curvature map $\kappa: \hat{M} \rightarrow \operatorname{Hom}\left(\wedge^{2}(\mathfrak{g}), \mathfrak{g}\right)$ of $\mathcal{P}$ differs from the curvature map of the initial Cartan geometry $(M, \hat{M}, \omega)$ by the constant bilinear form $[,]_{\mathfrak{g}}$, which explains that the derivatives (hence the Killing generators) of both curvature maps coincide.

7.0.2. Killing fields and distribution on $\hat{M} \times \mathbb{R}^{m}$. - The framing $\left(X_{1}, \ldots, X_{m}\right)$ identifies $T \hat{M}$ with the product $\hat{M} \times \mathbb{R}^{m}$ in the following way: every vector $\xi \in$ $T_{\hat{x}} \hat{M}$ writes $\xi_{1} X_{1}(\hat{x})+\ldots+\xi_{m} X_{m}(\hat{x})$, allowing to identify $\xi$ with $\left(\xi_{1}, \ldots, \xi_{m}\right) \in$ $\mathbb{R}^{m}$.

The local flows $\varphi_{X_{i}}^{t}$ induce local flows on the tangent bundle $T \hat{M}$, yielding vector fields $X_{i}^{*}, 1 \leq i \leq m$, on $\hat{M} \times \mathbb{R}^{m}$. Those fields have a simple expression involving $X_{i}$ and the curvature function (computations can be found in $[\mathrm{P}][$ Lemma 4.9]) :

$$
X_{i}^{*}(\hat{x}, u)=\left(X_{i}(\hat{x}), \kappa(\hat{x})\left(e_{i}, u\right)\right) \in T_{\hat{x}} \hat{M} \times \mathbb{R}^{m} .
$$

Let us call $\Delta$ the distribution on $\hat{M} \times \mathbb{R}^{m}$, defined at each $(\hat{x}, u)$ by $\Delta(\hat{x}, u)=$ $\operatorname{Span}\left(X_{1}^{*}(\hat{x}, u), \ldots, X_{m}^{*}(\hat{x}, u)\right)$. The distribution $\Delta$ is related to Killing fields as follows. The identification $T \hat{M} \simeq \hat{M} \times \mathbb{R}^{m}$ mentioned above allows to see every local vector field on $U \subset \hat{M}$ as a map $\varphi_{X}: U \rightarrow \mathbb{R}^{m}$. Assume that such a vector field satisfies $X(\hat{x})=u$. The condition that $X$ commutes with the fields $X_{i}^{\prime} s$ up to order 1 at $\hat{x}$, is equivalent to the graph of the map $\varphi_{X}$ being tangent to $\Delta(\hat{x}, u)$ at $(\hat{x}, u)$. We thus see that if there exists an integral manifold of $\Delta$ in the neighborhood of the point $(\hat{x}, u)$, then this manifold is locally the graph of a map $\varphi_{X}: U \subset \hat{M} \rightarrow \mathbb{R}^{m}$, associated to a Killing field $X$ on $U$, with $X(\hat{x})=u$ (see $[\mathrm{P}][$ Lemma 4.6$])$.

Observe that except in the case of a flat parallelism (namely when all the $X_{i}$ 's commute pairwise), the distribution $\Delta$ never satisfies the Frobenius integrability condition on $\hat{M} \times \mathbb{R}^{m}$. The best we can hope is to find a few integral leaves for $\Delta$.

7.0.3. Some useful submanifolds in $\hat{M} \times \mathbb{R}^{m}$. - We recall from Sections 2.1 and 2.2 the definition of the derivatives $D^{r} \kappa$, the general curvature map $\mathcal{D} \kappa$, and $\operatorname{Kill}^{r}(\hat{x})$, the set of Killing generators of order $r$ at $\hat{x}$. Recall that $\hat{M}^{\text {int }}$ is the dense open subset of $\hat{M}$ where the rank of $\mathcal{D} \kappa$ is locally constant.

We define for every $r \in \mathbb{N}^{*}$,

$$
F_{r}:=\left\{(\hat{x}, u) \in \hat{M} \times \mathbb{R}^{m} \mid u \in \operatorname{Kill}^{r}(\hat{x})\right\} .
$$


Those are submanifolds of $\hat{M} \times \mathbb{R}^{m}$, as well as

$$
\Sigma^{\mathrm{int}}:=\left(\hat{M}^{\mathrm{int}} \times \mathbb{R}^{m}\right) \cap F_{m+1} .
$$

By what was said before, proving Theorem 2.2 is equivalent to finding an integral leaf of $\Delta$ through each point of $\Sigma^{i n t}$. It is thus enough to show that $\Delta$ defines a distribution on $\Sigma^{\text {int }}$ satisfying Frobenius integrability condition. To do this, we first work on smaller manifolds, defining

$$
\Omega:=\left\{\hat{x} \in \hat{M} \mid \operatorname{dim}\left(\operatorname{Kill}^{j}\right), 1 \leq j \leq m+2 \text {, are locally constant around } \hat{x}\right\},
$$

and for every $r \in \mathbb{N}^{*}$,

$$
\begin{gathered}
U_{r}:=\left\{\hat{x} \in \Omega \mid \operatorname{dim}\left(\operatorname{Kill}^{r}(\hat{x})\right)=\operatorname{dim}\left(\operatorname{Kill}^{r+1}(\hat{x})\right)\right\}, \\
\Sigma_{r}=F_{r} \cap\left(U_{r} \times \mathbb{R}^{m}\right) .
\end{gathered}
$$

The first point is that $\Delta$ is tangent to the submanifolds $\Sigma_{r}$, as shows the following lemma.

Lemma 7.1 ([No], Lemma 12; compare Lemma 4.7 of [P])

For each $1 \leq r \leq m+1$, the submanifolds $\Sigma_{r}$ are stable by the local flows $\phi_{X_{i}^{*}}^{t}$. More precisely, if $(\hat{x}, u) \in \Sigma_{r}$, then for every $i=1, \ldots, n, X_{i}^{*}(x, u) \in T \Sigma_{r}$.

Next, because $F_{r} \subset F_{1}$, the distribution $\Delta$, seen as a distribution of $\Sigma_{r}$ satisfies the Frobenius condition, hence is integrable. This follows from

Lemma $7.2\left([\mathrm{P}]\right.$, Lemma 4.10). - At each $(\hat{x}, u) \in F_{1}$ and for every $1 \leq i \leq$ $j \leq m$, the brackets $\left[X_{i}^{*}(x, u), X_{j}^{*}(x, u)\right]$ belong to $\Delta(x, u)$.

From this integrability property, and the previous discussion, we infer that $\operatorname{dim} \operatorname{Kill}^{r}(\hat{x}) \geq \operatorname{dim} \mathfrak{k i r l}^{\text {loc }}(\hat{x})$ for all $\hat{x} \in U_{r}$. Since the reverse inequality always holds, we get for every $1 \leq r \leq m+1$ :

$$
\operatorname{Kill}^{r}(\hat{x})=\operatorname{Kill}^{m+1}(\hat{x})=\mathfrak{k i r l}^{\mathrm{loc}}(\hat{x}) .
$$

We conclude that for every $1 \leq r \leq m+1$, $\Sigma^{i n t} \cap\left(U_{r} \times \mathbb{R}^{m}\right)$ is an open subset of $\Sigma_{r}$.

We now conclude in the following way. The inequalities $\operatorname{dim}\left(\mathrm{Kill}^{j}\right) \geq$ $\operatorname{dim}\left(\right.$ Kill $\left.^{j+1}\right)$ show that at each point $\hat{x} \in \Omega$, there must exist $1 \leq r \leq m+1$ such that $\operatorname{dim}\left(\mathrm{Kill}^{r}\right)=\operatorname{dim}\left(\mathrm{Kill}^{r+1}\right)$. This implies that $\Sigma^{\text {int }} \cap\left(\Omega \times \mathbb{R}^{m}\right)$ is an open subset of $\bigcup_{r=1}^{m+1} \Sigma_{r}$. In particular, $\Delta$ is tangent to $\Sigma^{i n t} \cap\left(\Omega \times \mathbb{R}^{m}\right)$ and satisfies the Frobenius integrability condition there. Finally, because $\Sigma^{i n t} \cap\left(\Omega \times \mathbb{R}^{m}\right)$ is a dense open set of the manifold $\Sigma^{i n t}$, this property holds true on $\Sigma^{\text {int }}$. This yields Theorem 2.2. 


\section{Annex B: Closed orbits in representations of $\operatorname{PSL}(2, \mathbb{R})$}

We provide below a proof of Lemma 4.2.

Let us consider the basis of $\mathfrak{s l}(2, \mathbb{R})$ given by

$$
H=\left(\begin{array}{cc}
1 & 0 \\
0 & -1
\end{array}\right), E=\left(\begin{array}{ll}
0 & 1 \\
0 & 0
\end{array}\right) \text { and } F=\left(\begin{array}{ll}
0 & 0 \\
1 & 0
\end{array}\right) .
$$

All hyperbolic flows in $\operatorname{PSL}(2, \mathbb{R})$ are conjugated inside $\operatorname{PSL}(2, \mathbb{R})$, and the same is true for elliptic flows. Hence, we may assume that $\left\{\varphi^{t}\right\}$ is generated either by $H$, or by $E-F$.

We first do the proof when the representation is irreducible. We then know that up to isomorphism, $\rho$ is induced by the action of $\mathrm{SL}(2, \mathbb{R})$ on homogeneous polynomials in 2 variables, the action being by linear substitution. Observe that the action of $-I d \in \operatorname{PSL}(2, \mathbb{R})$ is trivial only when the polynomials have even degree, so that $V$ is odd dimensional. Let us put $2 k+1=\operatorname{dimV}$, and denote by $\bar{\rho}: \mathfrak{s l}(2, \mathbb{R}) \rightarrow \mathfrak{g l}(2 k+1, \mathbb{R})$ the representation induced by $\rho$ at the Lie algebra level. There is a suitable basis $e_{1}, \ldots, e_{2 k+1}$ of $V$ where

$$
\begin{gathered}
\bar{H}=\bar{\rho}(H)=\left(\begin{array}{cccccc}
2 k & & & & \\
& 2 k-2 & & & \\
& \ddots & & & \\
& & -2 k+2 & \\
& & & & -2 k
\end{array}\right) \\
\\
\end{gathered}
$$

Because $2 k+1$ is odd, it is easy to check that $\operatorname{Fix}\left(\varphi^{t}\right)$ consists of the line generated by $v$. Moreover, $\rho(\mathrm{SL}(2, \mathbb{R}))$ preserves a pseudo-Riemannian scalar product $g$ on $V$, having type $(k, k+1)$. Precisely, $g(x, x)=\left(\sum_{m=1}^{k} 2 a_{m} x_{m} x_{2 k+2-m}\right)+$ $a_{k+1} x_{k+1}^{2}$, where $a_{1}$ is any element of $\mathbb{R}^{*}$ and the $a_{i}$ 's satisfies the relations $a_{i+1}=\frac{2 k+1-i}{i} a_{i}$ for $i=1, \ldots, k$.

Let us check that $g(v, v) \neq 0$. When $\left\{\varphi^{t}\right\}$ is generated by $H$, it is obvious since $v$ is then on the line $\mathbb{R} . e_{k+1}$. Assume now that $\varphi^{t}$ is elliptic. If $g(v, v)=0$, then $v$ is included in the $g$-orthogonal $\{v\}^{\perp}$. By compactness of the 1-parameter group $\left\{\varphi^{t}\right\}$, we get a $\varphi^{t}$-invariant decomposition $v^{\perp}=L \oplus \mathbb{R} v$. Now, the dimension of $L$ is odd (namely $2 k-1$ ), hence $\varphi^{t}$ admits a line of fixed points in $L$. This gives a 2-plane of fixed points for $\varphi^{t}$ in $V$ : contradiction. 
We can conclude that the orbit $\mathcal{O}$ of $v$ under $\operatorname{PSL}(2, \mathbb{R})$ is closed in the following way. Because the action of $\operatorname{SL}(2, \mathbb{R})$ is algebraic, a non closed orbit must accumulate on some orbit of (strictly) smaller dimension. In our case, if not closed, the orbit $\mathcal{O}$ should have the origin in its closure, because there are no orbits of dimension 1 for finite dimensional representations of $\operatorname{PSL}(2, \mathbb{R})$. This is not possible, since $\mathcal{O}$ is included in the set $\{x \in V \mid g(x, x)=g(v, v)\}$ and $g(v, v) \neq 0$.

In the case $V$ is not irreducible, it splits as a direct sum $V=V_{1} \oplus \ldots \oplus V_{s}$ of irreducible representations. Let $u \in V$ having stabilizer $\left\{\varphi^{t}\right\}$, a hyperbolic or an elliptic flow. We write $u=u_{1}+\ldots+u_{s}$. In any finite dimensional irreducible representation of $\operatorname{PSL}(2, \mathbb{R})$, the stabilizer of a point is either $\operatorname{PSL}(2, \mathbb{R})$, a 1parameter group, or $\{i d\}$. Hence, the stabilizer of each $u_{i}$ is either $\operatorname{PSL}(2, \mathbb{R})$ or $\left\{\varphi^{t}\right\}$, because if other possibilities occured, the stabilizer of $u$ would be trivial. We assume this stabilizer is $\operatorname{PSL}(2, \mathbb{R})$ for $u_{1}, \ldots, u_{l}$ and $\left\{\varphi^{t}\right\}$ for $u_{l+1}, \ldots, u_{s}$. Let $\left(g_{k}\right)$ be a sequence in $\operatorname{PSL}(2, \mathbb{R})$ such that $g_{k}$. $u$ converges to $u_{\infty} \in V$. For each $i=l+1, \ldots, s$, we have $g_{k} \cdot u_{i} \rightarrow u_{i}^{\infty}$. Because $V_{i}$ is irreducible, we know by the first part of the proof that the orbit of $u_{i}$ under $\operatorname{PSL}(2, \mathbb{R})$ is closed. Hence there exists $\tilde{g}_{k}$ converging to $g_{\infty} \in \operatorname{PSL}(2, \mathbb{R})$ such that $\tilde{g}_{k} \cdot u_{l+1}=g_{k} \cdot u_{l+1}$. In particular $g_{k}=\tilde{g}_{k} \cdot \varphi^{t_{k}}$ for all $k$. But now, this implies $g_{k} \cdot u_{i}=\tilde{g}_{k} \cdot u_{i}$ for all $i=l+1, \ldots, s$, hence for all $i=1, \ldots, s$. We end up with $g_{k} \cdot u=\tilde{g}_{k} \cdot u$, so that $u_{\infty}=g_{\infty} . u$. This concludes the proof.

\section{Aknowledgments}

The author would like to thank the referee for making valuable suggestions to improve this text.

\section{BIBLIOGRAPHY}

[B] Y. Benoist, Orbites des structures géométriques rigides (daprès M. Gr omov). Progress in Math (1997), 1-17.

[BF] E.J. Benveniste, D. Fisher, Nonexistence of invariant rigid structures and invariant almost rigid structure, Comm. Anal. Geom. 13 (2005) 89-111.

[BFL] Y. Benoist, P. Foulon, F. Labourie, Flots d'Anosov à distributions stable et instable différentiables. J. Amer. Math. Soc. 5 (1992), no. 1, 33-74.

[CS] A. Čap, J. Slovák, Parabolic geometries. I. Background and general theory. Mathematical Surveys and Monographs, 154. American Mathematical Society, Providence, RI, 2009.

[D1] S. Dumitrescu, An invitation to quasihomogeneous rigid geometric structures. Bridging algebra, geometry, and topology, 107-123. 
[D2] S. Dumitrescu, Dynamique du pseudo-groupe des isométries locales sur une variété lorentzienne analytique de dimension 3. Ergodic Theory Dynam. Systems , 28 (2008), no. 4, 1091-1116.

[DG] S. Dumitrescu, A. Guillot, Quasihomogeneous analytic affine connections on surfaces. J. Topol. Anal. 5 (2013), no. 4, 491-532.

[DM] S. Dumitrescu, K. Melnick, Quasihomogeneous three-dimensional realanalytic Lorentz metrics do not exist. Geom. Dedicata 179 (2015), 229253.

[DZ] S. Dumitrescu, A. Zeghib, Géométries Lorentziennes de dimension 3: classification et complétude. Geom. Dedicata 149 (2010), 243-273.

[DaG] G. DAmbra, M. Gromov, Lectures on transformations groups: geometry and dynamics, Surveys in Differential Geometry (Cambridge), (1990), $19-111$.

[F] D. Fisher, Groups acting on manifolds: around the Zimmer program. In Geometry, rigidity, and group actions, Chicago Lectures in Math., pages 72-157. Univ. Chicago Press, Chicago, IL, 2011.

[FK] R. Feres, A. Katok, Ergodic theory and dynamics of G-spaces (with special emphasis on rigidity phenomena). Handbook of dynamical systems, Vol. 1A, 665-763, North-Holland, Amsterdam, 2002.

[Gr] M. Gromov, Rigid transformation groups, Geometrie Differentielle, (D. Bernard et Choquet-Bruhat Ed.), Travaux en cours, Hermann, Paris, 33, (1988), 65-141.

[Io] A. Iozzi, Invariant geometric structures: A non-linear extension of the Borel density theorem, Am. Jour. Math., 114 (1992), 627-648.

$[\mathrm{KN}]$ S. Kobayashi, K. Nomizu, Foundations of differential geometry I (New York : Interscience Publishers, 1963).

[M] K. Melnick, A Frobenius theorem for Cartan geometries, with applications. L'Enseignement Mathématique (Série II) 57 (2011), no. 1-2, 57-89.

[No] K. Nomizu, On local and global existence of Killing fields. Ann. of Math. 72 (1960), no. 2, 105-112.

[P] V. Pécastaing, On two theorems about local automorphisms of geometric structures. arXiv:1402.5048 [math.DG]. To appear in Annales de l'institut Fourier.

[Sh] R.W. Sharpe, Differential Geometry: Cartan's generalization of Klein's Erlangen Program. New York, Springer, 1997.

[Si] I. Singer, Infinitesimally homogeneous spaces. Comm. Pure Appl. Math. 13 (1960), 685-697.

[Z1] A. Zeghib, On Gromovs theory of rigid transformation groups: a dual approach. Ergodic Theory Dynam. Systems 20 (2000), no. 3, 935-946.

[Z2] A. Zeghib, Killing fields in compact Lorentz 3-manifolds, J. Differential Geom., 43, (1996), 859-894. 